\title{
Nonlinear Filtering Preserves Chaotic Synchronization via Master-Slave System
}

\author{
J. S. González-Salas, ${ }^{1}$ E. Campos-Cantón, ${ }^{2}$ F. C. Ordaz-Salazar, ${ }^{1}$ and E. Jiménez-López ${ }^{3}$ \\ ${ }^{1}$ Academia de Matemáticas, Universidad Politécnica de San Luis Potosí, Urbano Villalón 500, 78369 San Luis Potosí, SLP, Mexico \\ ${ }^{2}$ División de Matemáticas Aplicadas, Instituto Potosino de Investigación Científica y Tecnológica, \\ Camino a la Presa de San José 2055, 78216 San Luis Potosí, SLP, Mexico \\ ${ }^{3}$ Departamento de Físico Matemáticas, Universidad Autónoma de San Luis Potosí, Alvaro Obregón 64, \\ Col. Centro 78000 San Luis Potosí, SLP, Mexico \\ Correspondence should be addressed to E. Campos-Cantón; eric.campos@ipicyt.edu.mx
}

Received 17 December 2012; Revised 26 February 2013; Accepted 6 March 2013

Academic Editor: H. G. Enjieu Kadji

Copyright (C) 2013 J. S. González-Salas et al. This is an open access article distributed under the Creative Commons Attribution License, which permits unrestricted use, distribution, and reproduction in any medium, provided the original work is properly cited.

We present a study on a class of interconnected nonlinear systems and give some criteria for them to behave like a filter. Some chaotic systems present this kind of interconnected nonlinear structure, which enables the synchronization of a master-slave system. Interconnected nonlinear filters have been defined in terms of interconnected nonlinear systems. Furthermore, their behaviors have been studied numerically and theoretically on different input signals.

\section{Introduction}

In the study of nonlinear dynamics, much research was devoted to synchronization of chaotic systems, which have been unidirectional coupling [1], and one of the main applications is in secure communications systems [2]. Under unidirectional coupling, there is a master system that forces a slave system. Let us begin by considering a master system whose temporal evolution is ruled by the following equation:

$$
\dot{\mathbf{y}}=f(\mathbf{y}) \text {, }
$$

where $\mathbf{y} \in \mathbb{R}^{n}$ is the state vector, with $f$ defining a vector field $f: \mathbb{R}^{n} \rightarrow \mathbb{R}^{n}$. The slave system is given by

$$
\dot{\mathbf{x}}=g(\mathbf{x}, \mathbf{y}),
$$

where $\mathbf{x} \in \mathbb{R}^{m}$ is the state vector, and the function $g$ : $\mathbb{R}^{m} \times \mathbb{R}^{n} \rightarrow \mathbb{R}^{m}$ describes the dynamics of the slave system and coupling. The master system behaves as an autonomous system and the slave system is a forced system that under certain conditions could behave as a nonautonomous system, where its dynamic is completely determined by the master system; that is, it means that the slave system acts in function of the master system. Hence, the slave system can be seen as a driven system by an external force, and then it can be studied as a filter when the input signal comes from a master system or any external signal unidirectional coupled.

There are several asymptotical behaviors reported based on master-slave configuration. Some cases are summarized as follows: Identical Synchronization (IS) implies coincidence of the corresponding states of the interacting systems [1]. Lag Synchronization (LS) occurs when the trajectory of one oscillator is delayed by a specific time and is identical to the trajectory of the other oscillator [3]. Phase Synchronization (PS) means the lock of chaotic oscillator phases, regardless of their amplitudes [4]. Frequency Entrainment Synchronization (FES) occurs when two systems are oscillating with the same frequency [5]. Generalized Synchronization (GS) is defined as the presence of some functional relationship between the states of the slave and master systems [6]. Multimodal Generalized Synchronization (MGS) is presented when there are several basins of attraction for the slave system, and generalized synchronization is also presented $[7,8]$. The aforementioned chaotic synchronization phenomena tell us that some types of synchronization are stronger than others. Another characteristic of these asymptotic behaviors is that 
they always can be described in terms of a functional relationship. For example, let $\Phi$ be the GS function which relates the master system with the slave system; that is, $\mathbf{y}=$ $\Phi(\mathbf{x})$. In general, the way how synchronization phenomena can be detected is by means of the auxiliary method [6]; thus the way one can know that unidirectional coupled systems present GS is when they present asymptotic behavior:

$$
\lim _{t \rightarrow \infty}\left|\mathbf{x}^{1}(t)-\mathbf{x}^{2}(t)\right|=0,
$$

where $\mathbf{x}^{1}(t)$ and $\mathbf{x}^{2}(t)$ are solutions of the slave and auxiliary systems, respectively, initialized with different initial condition $\mathbf{x}^{1}(0) \neq \mathbf{x}^{2}(0)$. If this limit is satisfied, it indicates that the slave system has lost its sensitivity to initial conditions, thus losing their autonomy. Thence, the trajectory of the response system depends on the input signal from master system. If we consider the slave system as a forced system, then we will see that its behavior depends on an external forcing, like a filter does.

Forced Synchronization (FS) is defined as a phenomenon that occurs when oscillations of several chaotic systems $\mathbf{x}^{(1)}(t), \mathbf{x}^{(2)}(t), \ldots, \mathbf{x}^{(k)}(t)$ show correlated behavior because of an external signal applied to these oscillators [9]. Meanwhile, the conditions in order that a chaotic system behaves as a filter for an external signal under a specific coupling are given in [10] where this phenomenon was called nonlinear filtering (NF). So, these three phenomena (GS, FS, and NF) can be recognized when the limit (3) is satisfied and can be studied from the viewpoint of forced chaotic systems. GS contains NF and MGS because these two phenomena present asymptotic behavior. Nevertheless, there exists a difference between NF and MGS. For MGS, the initial conditions determine the basin of attraction where the trajectory of the slave system converges, the fact despite that there exists a functional relationship between the slave and the master systems, the slave system does not behave like a filter. For NF, the slave system must act in function of the external force and never in function of its initial conditions.

The dynamic of at least one state of several chaotic systems has similar structure to low pass filter, for example, the first equation of the Lorenz system $\dot{x}=-\sigma x+\sigma y$, where $x$ and $y$ can be seen as the output and input signals of a low pass linear filter, respectively. The second equation of the Lorenz system can be seen as a low pass nonlinear filter, $\dot{y}=-y+x(\rho-z)$, where $y$ is the output and $x$ and $z$ are the input signals of a low pass nonlinear filter. Without loss of generality, from the viewpoint of continuous dynamical systems, a filter can be seen as a forced dynamical system in which its response depends on its structure, given by their equations, and can be linear or nonlinear. Then a natural question would be the following: what is the effect on the response of forced nonlinear system given an input signal? In order to answer this question, we make a study of a chaotic system forced with different kinds of signals.

We are interested in explaining synchronization phenomenon of chaotic systems based on nonlinear interconnected structures, as these structures behave as a nonlinear filter that allows synchronization of master and slave systems. Thereby, the target is not to put forward a new nonlinear filter that can replace those designed with the specific purpose of preventing noise in the signal. Thus, the objective of this research is to show how the synchronization phenomenon underlies nonlinear interconnected structure. This kind of structure which is immersed in some chaotic systems could have some useful applications in filtering waves such as those originated from earthquakes and tsunamis, because they can be tuned to produce resonance at certain frequencies. So, the target of this work is to give features of the nonlinear filter as those given by the second and third equations of the Lorenz system in function of the parameters of the input signal $u$, which gives us a complementary perspective with respect to the analysis made in [9], where it was only proved and showed that a forced-chaotic system filters constant, sinus, and random signals. To achieve this goal, we make a study on the effect of nonlinear filter based on interconnected nonlinear systems for different input signals $u$, like sinusoidal, chaotic, and random signals. Several chaotic systems that have a similar structure to interconnected systems can be analyzed like a nonlinear filtering phenomenon.

The paper is organized as follows: Section 2 contains basic definitions of nonlinear filters based on $n$-interconnected systems; Section 3 presents a way how tuning the parameters of an $n$-interconnected system in order to the system behaves as a nonlinear filter; in Section 4 is the study of the response to amplitude and frequency of a nonlinear filter; Section 5 shows the response of the filter when the input signal is noise; Section 6 presents a study case of correlation coefficient analysis; in Section 7, we present the relation between nonlinear filters and chaotic systems; finally, conclusions are given in Section 8 .

\section{Nonlinear Filter Structure}

In the theory of linear systems, it is very well known that a first-order low pass linear filter is given as follows:

$$
\dot{x}_{1}=k_{1} x_{1}+k_{2} u,
$$

where $x_{1} \in \mathbb{R}$ is the output of the filter, and $k_{1}, k_{2} \in \mathbb{R}$ are parameters of the filter (4). These parameters control attenuation and amplitude of the input signal, respectively, and $u \in \mathbb{R}$ is the input signal to be filtered. Based on the configuration of low pass filter given by (4), a low pass nonlinear filter is defined as follows.

Definition 1. Let $x_{1} \in \mathbb{R}$ be an output signal, $u \in \mathbb{R}$ an input signal, and $k_{1}, k_{2}$, and $k_{3} \in \mathbb{R}$ parameters. Thus, a low pass nonlinear filter can be defined as follows:

$$
\dot{x}_{1}=k_{1} x_{1}+\left(k_{2}+k_{3} x_{1}\right) u .
$$

The parameter $k_{3}$ in the above definition is used to control the nonlinear term $x_{1} u$. Notice that when $k_{3}=0$, the nonlinear term disappears, $k_{3} x_{1} u$, and then the nonlinear filter (5) behaves as a linear filter. The structure of nonlinear filters (5) has been used to generate chaos, as it can be seen in the Lorenz system [11], but its states are interconnected. Therefore, a natural question emerges around nonlinear filtering: what are the characteristics of two nonlinear filters if 
they are interconnected and forced by the same signal $u$ ? An interconnected system via nonlinear filters is given as follows:

Definition 2. Let $x_{1}, x_{2} \in \mathbb{R}$ be output signals, $u \in \mathbb{R}$ an input signal, and $k_{1}, k_{2}, k_{3}, k_{4}, k_{5}$, and $k_{6} \in \mathbb{R}$ parameters. So, let us define an interconnected system via low pass nonlinear filters as follows:

$$
\begin{aligned}
& \dot{x}_{1}=k_{1} x_{1}+\left(k_{2}+k_{3} x_{2}\right) u, \\
& \dot{x}_{2}=k_{4} x_{2}+\left(k_{5}+k_{6} x_{1}\right) u .
\end{aligned}
$$

Thus the system (6) is called two-interconnected systems.

Now, in Definition 2, the parameters $k_{3}$ and $k_{6}$ are coupling parameters for the outputs $x_{1}$ and $x_{2}$, respectively. In general, an $n$-interconnected system can be defined by coupled $x_{n}$ to the output $x_{n+1}$ with $n>2$. This $n$ interconnected system has a core based on low pass linear filters $\dot{x}=K \mathbf{x}+B u$, where $\mathbf{x} \in \mathbb{R}^{n}$ is a state vector, $K \in$ $\mathbb{R}^{n \times n}$ is a matrix, and $B \in \mathbb{R}^{n \times 1}$ is a constant vector. Thus, we can define $n$-interconnected system with the following expression:

$$
\dot{x}=K \mathbf{x}+B u+f(\mathbf{x}, u),
$$

where the nonlinear function $f(\mathbf{x}, u) \in \mathbb{R}^{n}$ is constituted by terms $k_{i} x_{j} u, i \in\{1,2, \ldots, 3 n\}, j \in\{1,2, \ldots, n\}$. An $n$ interconnected system (7) is studied in [12] where the authors found that its model produces hyperbolic chaos when it is forced by a sinusoidal wave. On the other hand, system (7) is formed by a linear part $K \mathbf{x}$, an input signal $B u$, and a nonlinear part $f(\mathbf{x}, u)$, which can induce sensitivity to initial conditions $\left(x_{1}(0), x_{2}(0), \ldots, x_{n}(0)\right)$. But a filter must act in function of the input signal and not in function of the initial condition. Therefore, it is important to find conditions in order to guarantee that an interconnected system behaves as a filter. Roughly speaking, the response of a filter only depends on the kind of input signal and if the interconnected systems depend on initial condition, this is classified as generalized forced synchronization phenomenon by forced systems [9]. Therefore, according to context, a nonlinear filter is defined in the next way.

Definition 3. Let $\mathbf{x}^{i}(t)=\left(x_{1}^{i}(t), x_{2}^{i}(t), \ldots, x_{n}^{i}(t)\right)^{T}$ be a vector of output signals given by $n$-interconnected system (7) with initial condition $\mathbf{x}^{i}(0)=\left(x_{1}^{i}(0), x_{2}^{i}(0), \ldots, x_{n}^{i}(0)\right)^{T}$. System (7) is called an $n$-interconnected nonlinear filter if it always presents asymptotic behavior:

$$
\lim _{t \rightarrow \infty}\left|\mathbf{x}^{(1)}(t)-\mathbf{x}^{(2)}(t)\right|=0 .
$$

In Definition 3, if $t \rightarrow \infty$ and the output of the $n$ interconnected system is independent of the initial conditions, then system (7) is considered an $n$-interconnected nonlinear filter.

\section{Tuning of the Parameters}

We select the entries of the matrices $K$ and $B$ in order for condition (8) to be satisfied. Let us start by considering $\boldsymbol{\delta}(t)=\mathbf{x}^{(1)}(t)-\mathbf{x}^{(2)}(t)$, and then $\dot{\delta}(t)=\dot{\mathbf{x}}^{(1)}(t)-\dot{\mathbf{x}}^{(2)}(t)$. Now, by using (7) we deduce that $\dot{\boldsymbol{\delta}}=K \boldsymbol{\delta}(t)+f\left(\mathbf{x}^{(1)}, u\right)-f\left(\mathbf{x}^{(2)}, u\right)$ which can be rewritten as follows:

$$
\begin{aligned}
& \boldsymbol{\delta}(t)=\boldsymbol{\delta}(0) e^{K t}+e^{K t} \int_{0}^{t} e^{K s}\left(f\left(\mathbf{x}^{(2)}, u\right)\right. \\
& \left.-f\left(\mathbf{x}^{(1)}, u\right)\right) d s
\end{aligned}
$$

In order to describe the asymptotic behavior of (9), we state the following theorem.

Theorem 4. If system (7), forced by signal $u$, satisfyies the following conditions:

(1) there exists a positive constant $k$ such that $\operatorname{Re}\{\lambda\} \leq-k$, for every eigenvalue $\lambda$ of the linear part of system (7);

(2) $f(\mathbf{x}, u)$ is a continuous Lipschitz; that is, there exists a positive function $C[u]<\infty$ such that $\mid f\left(\mathbf{x}^{(2)}, u\right)-$ $f\left(\mathbf{x}^{(1)}, u\right)|\leq C[u]| \mathbf{x}^{(2)}-\mathbf{x}^{(1)} \mid$,

then, system (7) is a filter for $u$, provided that $k>\overline{C[u]}$, where

$$
\overline{C[u]}:=\lim _{t \rightarrow \infty} \frac{1}{t} \int_{0}^{t} C[u(s)] d s .
$$

Proof. Let $\mathbf{x}^{(1)}(t), \mathbf{x}^{(2)}(t)$, and $\boldsymbol{\delta}(t)$ defined be before. We want to prove that $k>\overline{C[u]}$ implies $\lim _{t \rightarrow \infty}|\boldsymbol{\delta}(t)|=0$. From (9) we have the following estimate:

$$
\begin{aligned}
|\boldsymbol{\delta}(t)| \leq & \left\|e^{K t}\right\||\boldsymbol{\delta}(0)|+\left\|e^{K t}\right\| \\
& \times \int_{0}^{t}\left\|e^{K(-s)}\right\|\left|f\left(\mathbf{x}^{(2)}, u\right)-f\left(\mathbf{x}^{(1)}, u\right)\right| d s \\
\leq & e^{\|K\||t|}|\boldsymbol{\delta}(0)|+e^{\|K\||t|} \\
& \times \int_{0}^{t} e^{\|K\||-s|}\left|f\left(\mathbf{x}^{(2)}, u\right)-f\left(\mathbf{x}^{(1)}, u\right)\right| d s .
\end{aligned}
$$

Using hypothesis (1), we obtain from the previous inequality the following estimate:

$$
\begin{aligned}
|\boldsymbol{\delta}(t)| \leq & e^{-k t}|\boldsymbol{\delta}(0)| \\
& +e^{-k t} \int_{0}^{t} e^{k s}\left|f\left(\mathbf{x}^{(2)}, u\right)-f\left(\mathbf{x}^{(1)}, u\right)\right| d s .
\end{aligned}
$$

If we multiply by $e^{k t}$, the last expression results in

$$
e^{k t}|\boldsymbol{\delta}(t)| \leq|\boldsymbol{\delta}(0)|+\int_{0}^{t} e^{k s}\left|f\left(\mathbf{x}^{(2)}, u\right)-f\left(\mathbf{x}^{(1)}, u\right)\right| d s .
$$

Using the hypothesis (2), it follows that

$$
e^{k t}|\boldsymbol{\delta}(t)| \leq|\boldsymbol{\delta}(0)|+\int_{0}^{t} e^{k s} C[u]|\boldsymbol{\delta}(s)| d s .
$$

Application of Gronwall's inequality yields

$$
|\boldsymbol{\delta}(t)| \leq|\boldsymbol{\delta}(0)| e^{-t\left(k-(1 / t) \int_{0}^{t} C[u(s)] d s\right)},
$$

which proves the theorem. 


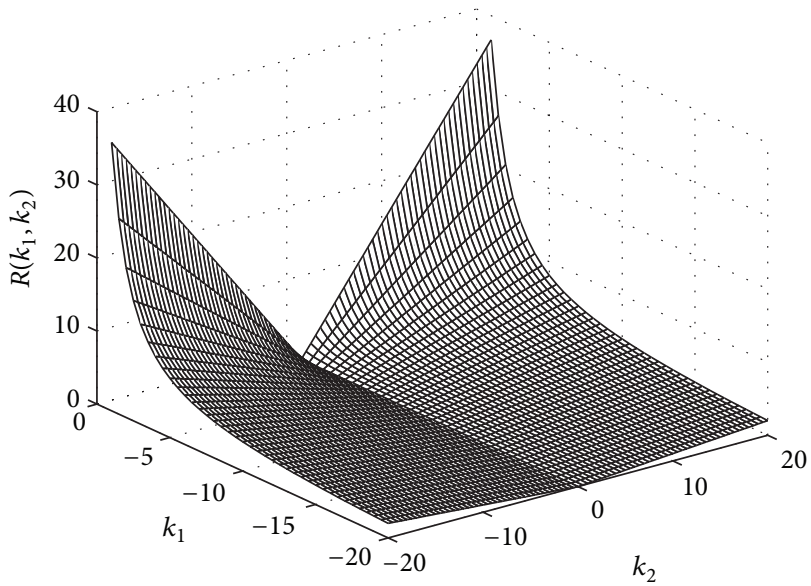

(a)

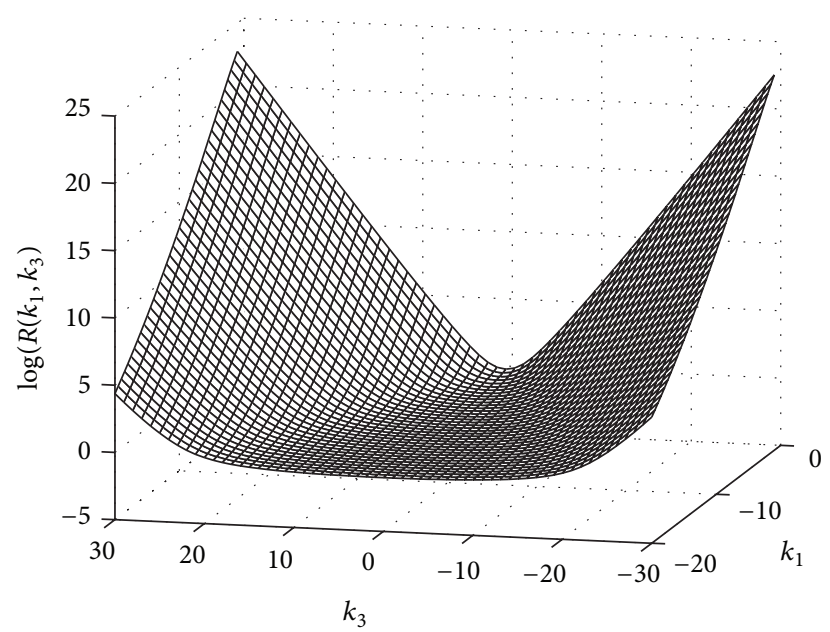

(b)

FIGURE 1: The maximal range of values of the filter (5) in function of its parameters when it is forced by $u=\sin t:(\mathrm{a}) R\left(k_{1}, k_{2}\right)$ and (b) $R\left(k_{1}, k_{3}\right)$.

We can infer from the above theorem that all elements of the matrix $K$ must be negative; that is, $k_{i}<0$ for $i=1, \ldots, n$. On the other hand, due to the fact that (9) does not depend on matrix $B$, then its terms are not conditioned and they can take any real value. These will be carried out in the remainder of this paper.

3.1. A Low Pass Nonlinear Filter. The analyzed case is the one-interconnected system given by (7); this case is confined to the particular case of the filter given by (5); where its elements are $x=x_{1} \in \mathbb{R}, A_{1 \times 1}=k_{1}, B_{1 \times 1}=k_{2}$, and $f\left(x_{1}, u\right)=u k_{3}$. According to the first condition of the above theorem, we have $k_{1}<0$, and now considering the nonlinear part of the filter, if the Lipschitz condition is satisfied when $\left|f\left(x_{1}\right)^{(1)}-f\left(x_{1}\right)^{(2)}\right| \leq\left|k_{3}\right||u||\delta(t)|$, giving as a result $c[u]=$ $\left|k_{3}\right||u|$, therefore the parameter $k_{3}$ can be any real value. Now, the interest is that the one-interconnected system behaves as a nonlinear filter; then Theorem 4 needs to be satisfied by $k_{1}<-\left|k_{3}\right| \overline{|u|}$. If the input signal is a constant, $u=\mu(\mu \in Z)$ and $\mu \leq-k_{1} /\left|k_{3}\right|$, then the response of the filter converges to zero; otherwise it diverges. Another case is when the input signal is $u=\mu \sin (\omega t)$, we have the following:

$$
\begin{aligned}
|\delta(t)| & \leq|\delta(0)| e^{k_{1} t+\left|k_{3}\right| \int_{0}^{t}|\mu \sin (\omega s)| d s} \\
& \leq|\delta(0)| e^{k_{1} t+\left|k_{3}\right||\mu| \int_{0}^{t} d s},
\end{aligned}
$$

which converges $\mu \leq-k_{1} /\left|k_{3}\right|$. Generally, condition (8) is always satisfied for oscillating functions $u$ with $|u(t)| \leq M$. However, for the case that $u$ is a polynomial of grade greater than 1 , that is, $u=a_{n} t^{n}+a_{n-1} t^{n-1}+\cdots+a_{0}$ for $n>1$, condition (8) is not satisfied.

Due to the fact that our interest is to tune the value of the parameters $k_{1}, k_{2}$, and $k_{3}$, we make a numerical study on the effect of these parameters on the response of $x_{1}$ when the oneinterconnected system (7) is being forced by the sinusoidal input signal $u=\sin (t)$. After a transient time, we calculate the maximal range of values of the one-interconnected system (7) by obtaining $R\left(k_{i}, k_{j}\right)=\operatorname{MAX}\left\{x_{1}(t)\right\}-\operatorname{MIN}\left\{x_{1}(t)\right\}(i \neq j)$, where $\operatorname{MAX}\left\{x_{1}(t)\right\}$ and $\operatorname{MIN}\left\{x_{1}(t)\right\}$ are the maximum and minimum values, respectively, of the response time series $x_{1}(t)$ in function of the parameters $k_{i}, k_{j}$. For example, if we want to calculate $R\left(k_{1}, k_{2}\right)$, then we fix the value of $k_{3}$ and simulate different time series of the one-interconnected system (7) for different values of $k_{1}, k_{2}$. In general, the value of $R\left(k_{i}, k_{j}\right)$ is calculated in function of the parameters which are varied and in each case condition (8) is verified if it is satisfied. Figures 1(a) and 1(b) show the graphs of $R\left(k_{1}, k_{2}\right)$ for $k_{3}=-1$ and $R\left(k_{1}, k_{3}\right)$ for $k_{2}=1$, respectively. These graphs show that the one-interconnected system (7) increments exponentially the amplitude of its response when the parameter $k_{3}$ increases its magnitude; meanwhile, the amplitude of $x_{1}$ is mildly incremented in a linear rate in function of the magnitude of the parameter $k_{2}$.

3.2. Interconnected Nonlinear Filters. Now, the first case is a two-interconnected system (6); the matrices $K_{2 \times 2}, B_{2 \times 1}$, and the nonlinear function $f(\mathbf{x}, u): \mathbb{R}^{2} \times \mathbb{R} \rightarrow \mathbb{R}^{2}$ are assumed as follows:

$$
\begin{aligned}
K & =\left(\begin{array}{cc}
k_{1} & 0 \\
0 & k_{4}
\end{array}\right), \quad B=\left(\begin{array}{l}
k_{2} \\
k_{5}
\end{array}\right), \\
f(\mathbf{x}, u) & =\left(\begin{array}{l}
k_{3} u x_{2} \\
k_{6} u x_{1}
\end{array}\right) .
\end{aligned}
$$

System (6) is asked to behave as a nonlinear filter, so the conditions of Theorem 4 are used to select the value of the parameters $\left\{k_{i}\right\}$. From the first condition of the theorem, we realize that $k_{1}, k_{4}<0,\left\{k_{2}, k_{5}\right\} \in \mathbb{R}$, and if $A>B \int_{0}^{t}|u| d s$, where $A=\max \left\{k_{1}, k_{4}\right\}$ and $B=\max \left\{k_{3}, k_{6}\right\}$, then system (6) is a filter for the signal $u$. In order to know more about the characteristics of the values of the parameters, we calculate 


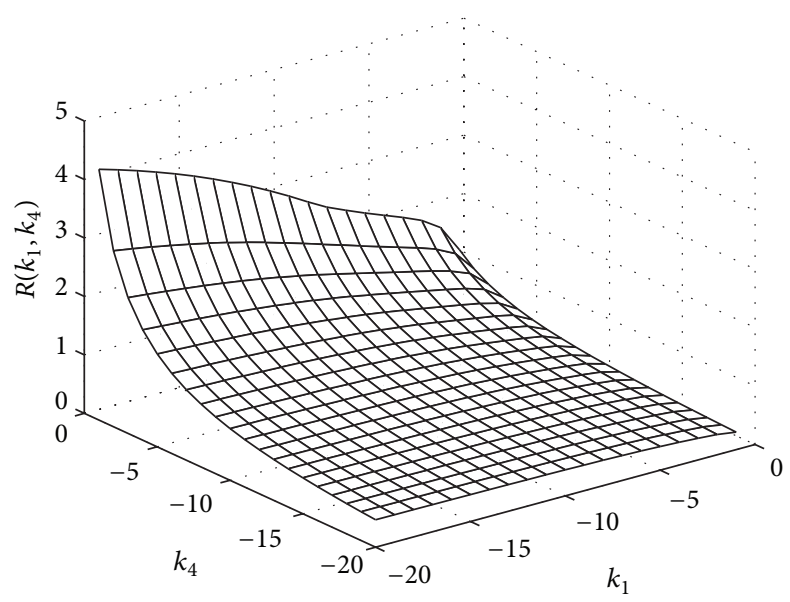

(a)

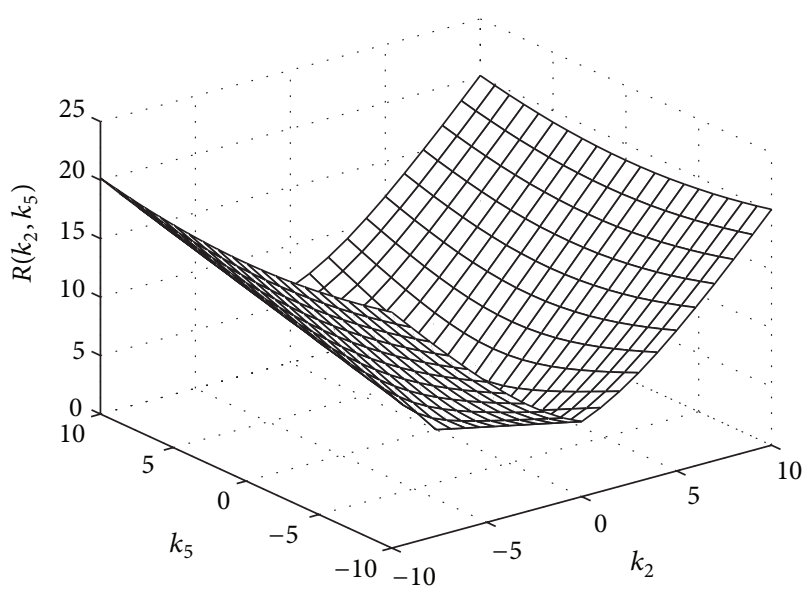

(b)

Figure 2: The maximal range of values of the filter (17) in function of its parameters when the filter is forced by $u=\sin t$. (a) $R\left(k_{1}, k_{4}\right)$ and (b) $R\left(k_{2}, k_{5}\right)$.

the eigenvalues of the Jacobian of system (6), which results in the following:

$$
\begin{aligned}
\lambda_{1,2}=\frac{1}{2} & \left(k_{1}+k_{4}\right. \\
& \left. \pm \sqrt{\left(k_{1}+k_{4}\right)^{2}-4\left(k_{1} k_{4}-k_{3} k_{6} u^{2}\right)}\right) .
\end{aligned}
$$

Our purpose is that system (6) converges; then $\operatorname{Re}\left\{\lambda_{i}\right\}$ must be negative. Therefore, we need to guarantee that $k_{1} k_{4} \geq$ $k_{3} k_{6} u^{2}$ is true. Due to $u^{2} \geq 0$, then the parameters $k_{3}$ and $k_{6}$ need to be opposite sign, that is, either $k_{3}<0, k_{5}>0$ or $k_{3}>0, k_{6}<0$. We choose arbitrarily the first relation of signs.

The effect of the parameters $\left\{k_{i}\right\}$ on the response $x$ of the interconnected nonlinear filters is computed by $R\left(k_{i}, k_{j}\right)$ when system (6) is forced by $u=\sin (t)$. Several calculations were made and for each of them we verify that the nonlinear filtering condition (8) was satisfied. Figure 2(a) shows $R\left(k_{1}, k_{4}\right)$ when the parameters of the filter (6) are fixed to $k_{2}=k_{5}=k_{6}=1, k_{3}=-1$. Figure $2(\mathrm{~b})$ shows $R\left(k_{2}, k_{5}\right)$ for $k_{1}=k_{4}=k_{3}=-1$ and $k_{6}=1$.

In the remainder of this paper, we studied the effect of the parameters input signal $u$ on the response of the coupled filter (6); that is, we tune the parameters of the filter, vary the parameter's values of the input signal, and observe the effect of the response. Therefore, we need to fix $k_{i}(i=1, \ldots, 6)$. Our interest is focus on the study of the response to the input $u$ when the linear filters $x_{1}, x_{2}$ are coupled in a nonlinear way. Without loss of generality and seeking clarity in our study, we consider $k_{5}=0$. The rest of the parameter values could vary in the intervals $k_{1}<0, k_{4}<0, k_{3} k_{6}<0,\left\{k_{2}, k_{5}\right\} \in \mathbb{R}$, which is a rich variety of values where the filter works. We can select any value in these intervals and produce similar responses; nevertheless, we fix the values to $k_{1}=-1, k_{2}=28, k_{3}=-1$, $k_{4}=-2.66, k_{5}=1$, which will be used in the rest of the paper.

\section{Response to the Amplitude and Frequency}

In this section, we present a study on the effect of the filter's response (6) as function of parameter's values of the sinusoidal input signal $u(t)=\mu \sin (\omega t)$. Figure 3(a) shows the output signals of the $n$-interconnected nonlinear filter for the cases $\mu=1, \omega=3$ and $\mu=1, \omega=5$. One can see that the orbits have a form of a Lissajous curve and the amplitude of the output signal as the frequency of the input signal is increased. In general, to see the effect of amplitude $\mu$ on the filter's response, we calculate the length of the Lissajous $L$ in this way

$$
L=\int_{0}^{T} \sqrt{\left(\dot{x}_{1}\right)^{2}+\left(\dot{x}_{2}\right)^{2}} d t
$$

where $T$ is the period of the orbit $\left(x_{1}(t), x_{2}(t)\right)$. The effect of the input signal's frequency $\omega$ on the length $L$ of the orbit of the filter's response is shown in Figure 3(b). Thus, $L$ falls exponentially according to the increment of the frequency $\omega$. System (6) presents a rejection to high frequencies because it is a low pass filter.

Now, fixing the frequency to $\omega=1.0$ and varying the amplitude $\mu$, we observe that new frequency components appear in the Fourier Transform (FT) of the output signal $x_{1}$. When the amplitude increases, then more peaks of frequency appear as multiples of the input signal's frequency. As is shown in Figures 4(a) and 4(b), $\mu=1$ and 5, respectively. This is a characteristic of a nonlinear filter that linear filters do not present.

The nonlinear filters display assorted behaviors, that is, contrary to the example shown in Figure 4, where the amplitude is fixed to $\mu=10$ and frequency $\omega$ is varied. Figures 5(a) and 5(b) show the FT of the response of the filter (6) for $\omega=1$ and $\omega=2$, respectively. Comparing both plots of Figure 5, we see that frequency peaks appear at multiples of input signal's frequency $\omega$. 


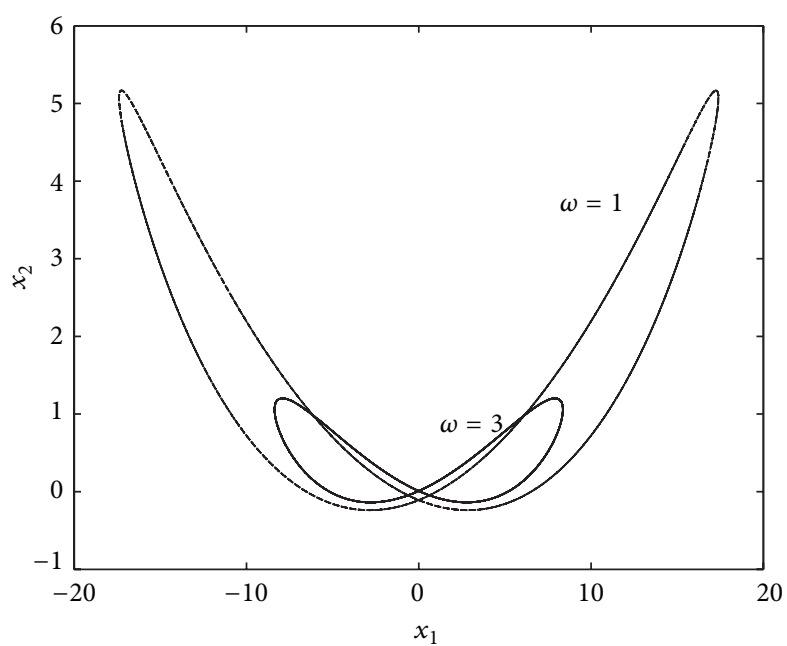

(a)

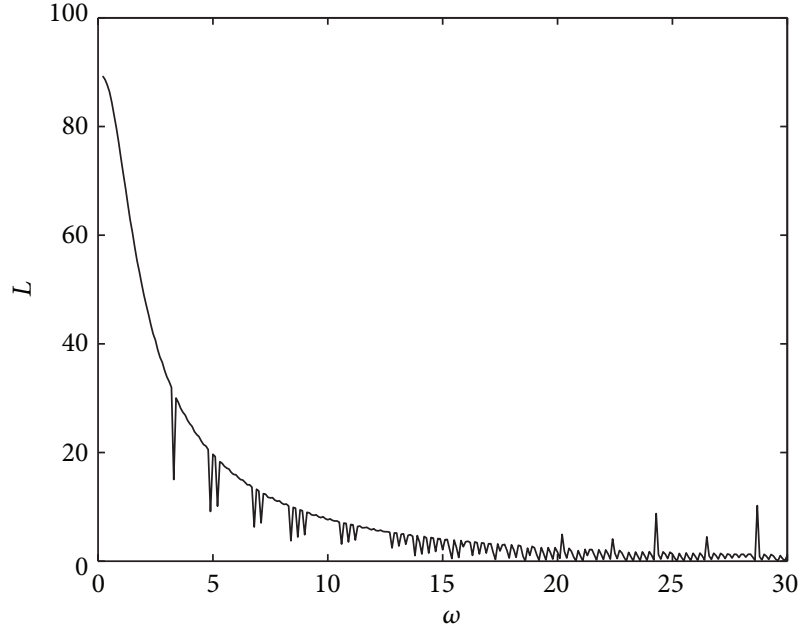

(b)

FIGURE 3: (a) The orbits of two Lissajous curves calculated by forcing system (6) with $\mu=1$ and $\omega=1$, 3. (b) We can see how the size of the Lissajous curve $L$ falls exponentially, as $\omega$ increments its value.

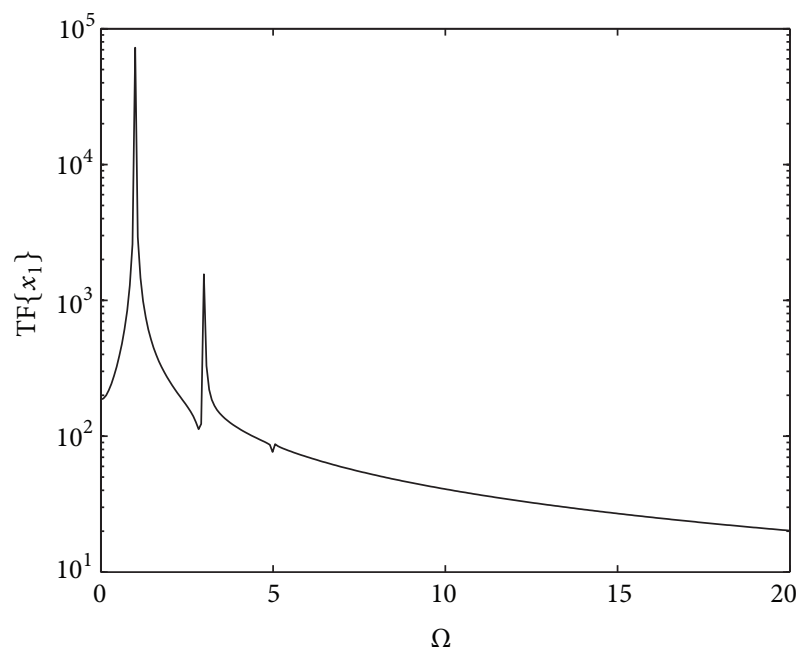

(a)

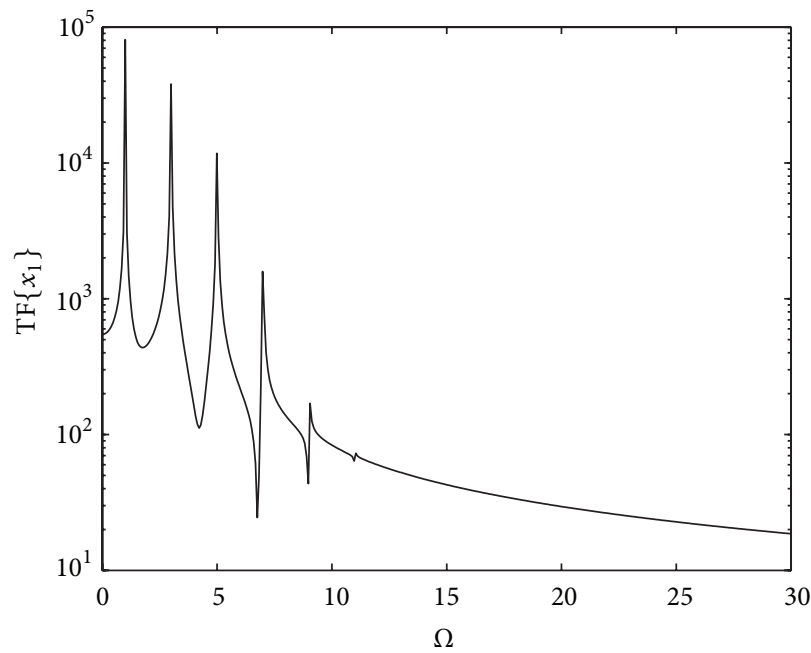

(b)

FIGURE 4: Both plots are the FT for the state $x_{1}$ of the filter (6) when it is filtering the input signal $u=\mu \sin (t)$ for the cases (a) $\mu=1.0$ and (b) $\mu=5$. These graphs show that when $\mu$ is incremented, new component frequency peaks appear in the power spectrum of the filter's response.

4.1. A Theoretical Justification. For the purpose of giving a theoretical justification of the behavior of the filter (6) when it is forced by the sinusoidal signals, we can see in the interconnected nonlinear filter (6) that $\dot{x}_{1}$ and $\dot{x}_{2}$ are linear first-order differential equations which can be rewritten in the next form:

$$
\begin{array}{r}
x_{1}(t)=\mu e^{k_{1} t}\left(k_{2} \int e^{-k_{1} t} \sin (\omega t) d t\right. \\
\left.+k_{4} \int e^{-k_{1} t} \sin (\omega t) x_{2}(t) d t\right), \\
x_{2}(t)=k_{6} \mu e^{k_{5} t} \int e^{-k_{5} t} \sin (\omega t) x_{1}(t) d t .
\end{array}
$$

Without loss of generality and for seeking clarity, we consider the parameter $k_{5}=0$. Applying integration by parts to (20) and considering that the states $x_{1}(t)$ and $x_{2}(t)$ are functions that depend on time and frequency and that $\mu$ is a constant, we have the following:

$$
\begin{array}{r}
x_{1}(t)=\frac{-\mu}{\omega^{2}+k_{1}^{2}}\left(k_{2}\left(k_{1} \sin (\omega t)+\omega \cos (\omega t)\right)\right. \\
+k_{3}\left(k_{1} \sin (\omega t)+\omega \cos (\omega t) x_{2}(t)\right) \\
-k_{4} e^{k_{1} t} \int e^{-k_{1} t}\left(k_{1} \sin (\omega t)\right. \\
\left.+\omega \cos (\omega t)) \dot{x}_{2} d t\right) .
\end{array}
$$




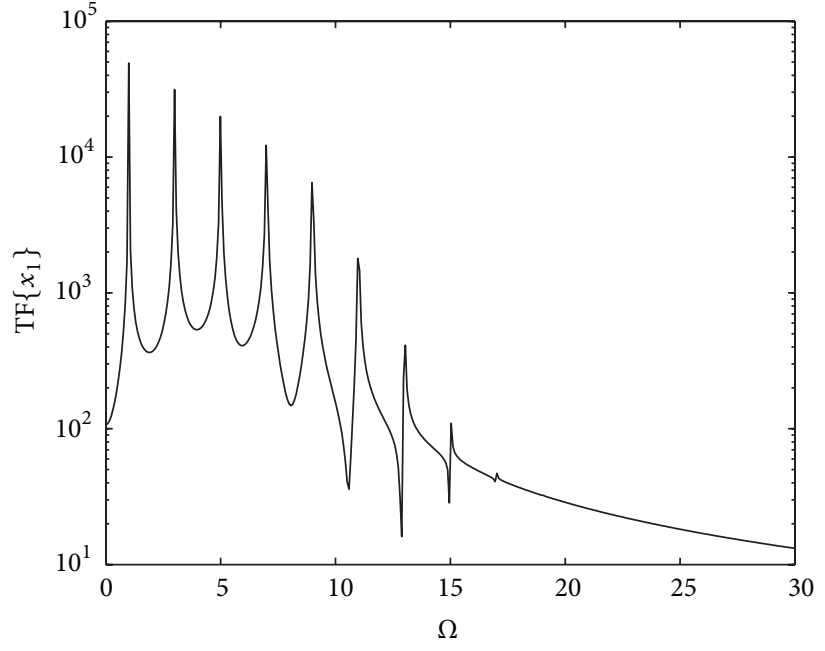

(a)

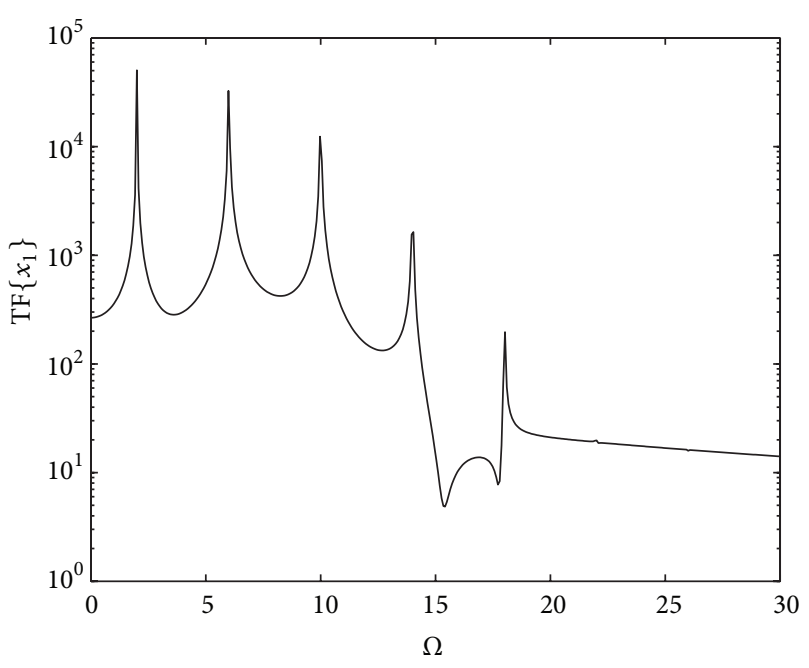

(b)

FIGURE 5: Both plots are the FT of the state $x_{1}$ of the filter (6) when it is filtering the input signal $u=10 \sin (\omega t)$ for the cases (a) $\omega=1$ and (b) $\omega=2$.

And for (21) we have

$$
\begin{aligned}
& x_{2}(t)=\frac{-\mu k_{6}}{\omega^{2}+k_{5}^{2}}\left(\left(k_{5} \sin (\omega t)+\omega \cos (\omega t)\right) x_{1}(t)\right. \\
& -e^{k_{5} t} \int e^{-k_{5} t}\left(k_{5} \sin (\omega t)\right. \\
& \left.+\omega \cos (\omega t)) \dot{x}_{1} d t\right) \text {. }
\end{aligned}
$$

Note that in (22) and (23), the magnitude values of $x_{1}(t)$ and $x_{2}(t)$ decrease when the frequency $\omega$ increases. Furthermore, since the terms

$$
\begin{aligned}
& e^{k_{1} t} \int e^{-k_{1} t}\left(k_{1} \sin (\omega t)+\omega \cos (\omega t)\right) \dot{x}_{2} d t \\
& e^{k_{5} t} \int e^{-k_{5} t}\left(k_{5} \sin (\omega t)+\omega \cos (\omega t)\right) \dot{x}_{1} d t
\end{aligned}
$$

have factors $\left(\omega^{2}+k_{5}^{2}\right)^{n},\left(\omega^{2}+k_{1}^{2}\right)^{n}$ for $n \leq-2$, then we approximate (22) and (23) to

$$
\begin{gathered}
x_{1}(t) \approx-\frac{\mu}{\omega^{2}+k_{1}^{2}}\left(k_{2} G_{1}(t)+k_{3} G_{1}(t) x_{2}(t)\right), \\
x_{2}(t) \approx-\frac{\mu k_{6}}{\omega^{2}+k_{5}^{2}} G_{2}(t) x_{1}(t),
\end{gathered}
$$

where $G_{1}(t)=k_{1} \sin (\omega t)+\omega \cos (\omega t), G_{2}(t)=k_{5} \sin (\omega t)+$ $\omega \cos (\omega t)$ are periodic functions with period given by the input signal. Substituting (25) in (26), we have

$$
x_{1}(t) \approx-\frac{\mu k_{2} G_{1}(t)}{\omega^{2}+k_{1}^{2}}+\frac{\mu^{2} k_{4} k_{8} G_{1}(t) G_{2}(t)}{\left(\omega^{2}+k_{1}^{2}\right)\left(\omega^{2}+k_{5}^{2}\right)} x_{1}(t) .
$$

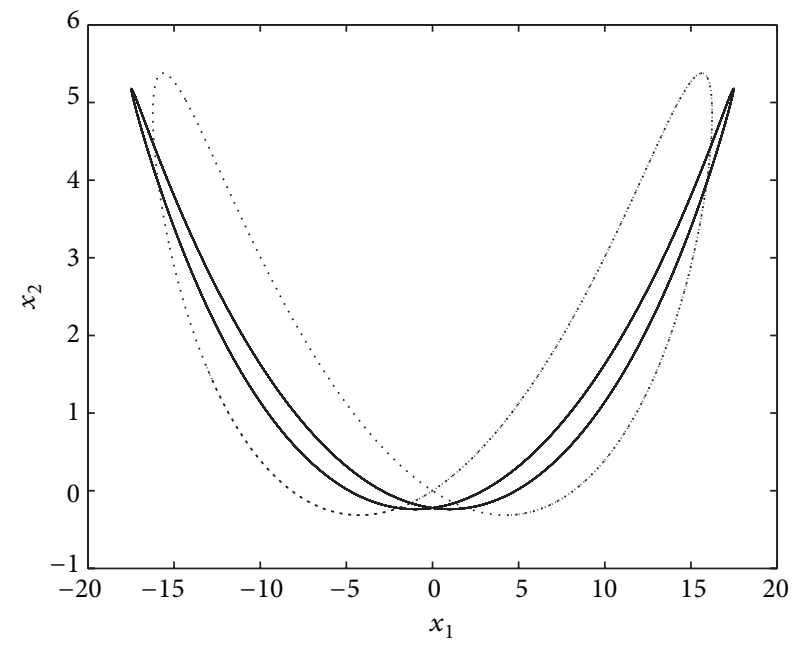

Figure 6: Comparison of Lissajouses. Lissajous with solid line corresponds to the solution of system (6) and Lissajous with dashed line corresponds to its approximated solution plotted with the parametric equations (28) and (29).

Now, solving for $x_{1}(t)$ from (27), it results that

$$
x_{1}(t) \approx \frac{-\mu k_{2}\left(\omega^{2}+k_{5}^{2}\right) G_{1}(t)}{\omega^{4}+\left(k_{1}^{2}+k_{5}^{2}\right) \omega^{2}+k_{1}^{2} k_{5}^{2}-\mu^{2} k_{3} k_{6} G_{1}(t) G_{2}(t)}
$$

And for $x_{2}(t)$ it results that

$$
x_{2}(t) \approx \frac{\mu^{2} k_{2} k_{6} G_{1}(t) G_{2}(t)}{\omega^{4}+\left(k_{5}^{2}+1\right) \omega^{2}+k_{5}^{2}-\mu^{2} k_{3} k_{6} G_{1}(t) G_{2}(t)} .
$$

Figure 6 shows the orbit of the solution of (28) and (29) and the orbit of the numerical solution of the filter (6) when 


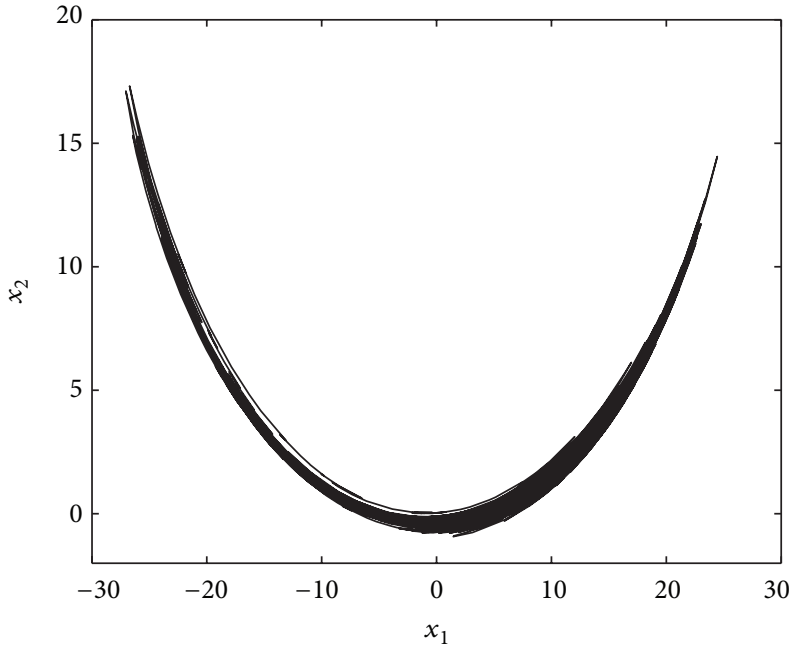

(a)

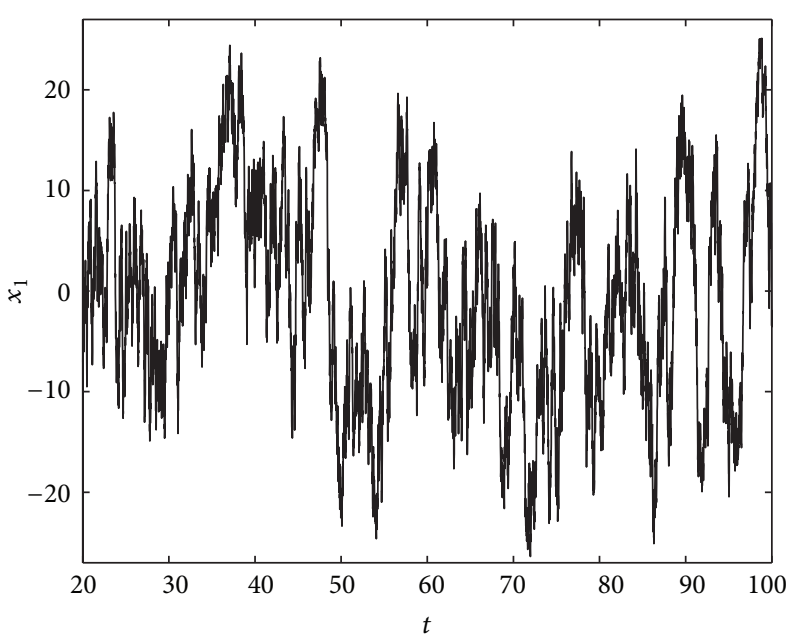

(b)

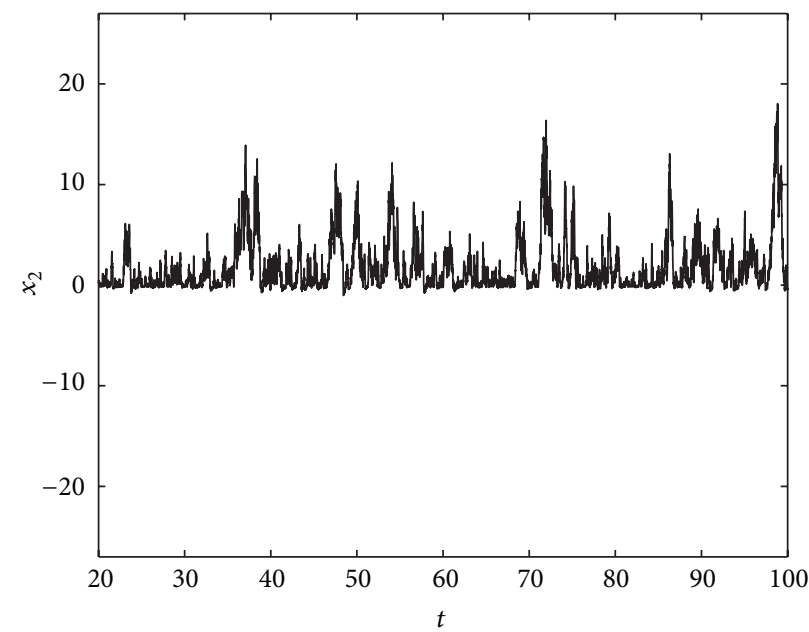

(c)

FIGURE 7: Plots of the filter's response (6) when a random input signal $u=10 \eta(t)$ is filtered. (a) The phase space $\left(x_{1}, x_{2}\right)$. The time series (b) $x_{1}(t)$ and $(\mathrm{c}) x_{2}(t)$.

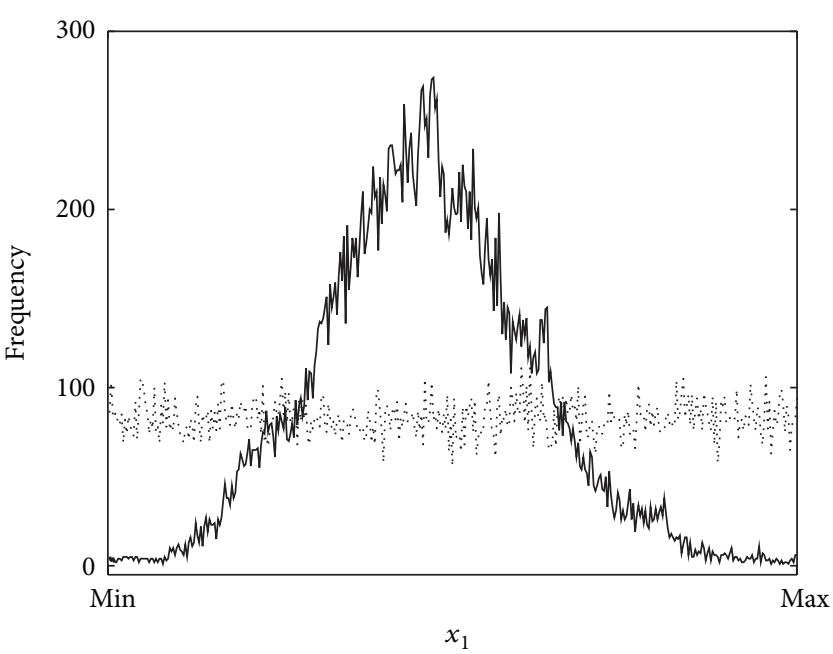

(a)

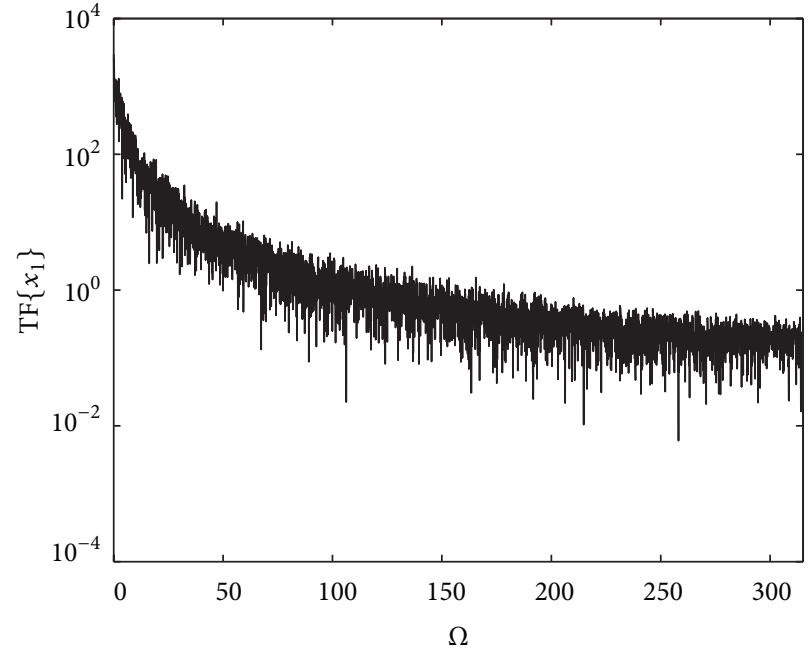

(b)

FiguRE 8: Response of the filter (6) when it is forced by the random signal $u=\eta(t)$. (a) Graphs of the histograms of the state $x_{1}$ (continuous line) and histogram of the external input signal $u$ (dotted line). (b) TF of the component $x_{1}$. 


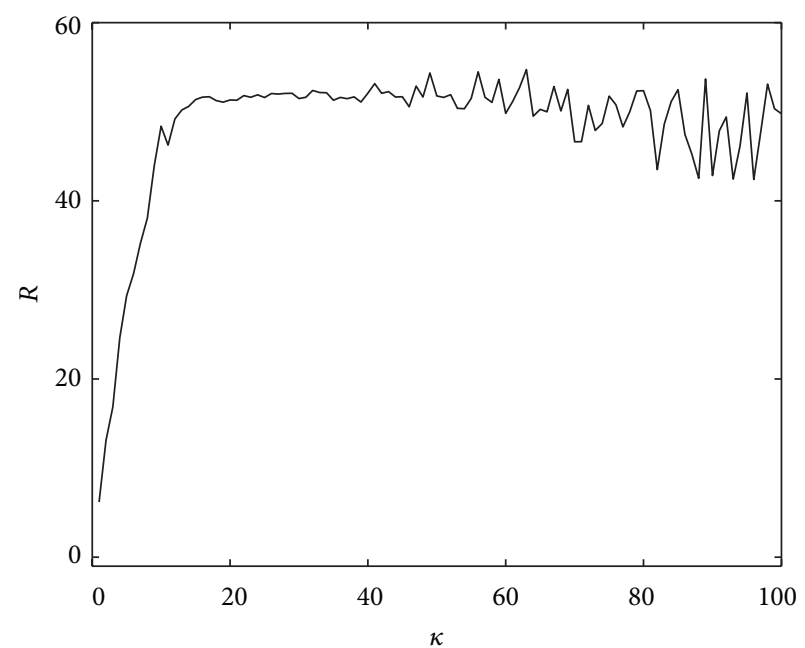

FIGURE 9: Range of values of the state $x_{1}$ in function of the $\kappa$ when system (6) filters random signal.

$\mu=1.0$ and $\omega=1.0$. The thin Lissajous curve is calculated with (6) and another with (28) and (29). We can see that both orbits oscillate in a Lissajous curve of one knot and the same symmetric form with respect to the $y$-axis, but slightly different amplitude.

Now, we analyze the FT of the equations of system (6) which are given as follows:

$$
\begin{gathered}
j \Omega X_{1}(\Omega)=k_{1} X_{1}(\Omega)+k_{2} \operatorname{FT}\{u\}+k_{3} \operatorname{FT}\left\{u x_{2}\right\}, \\
j \Omega X_{2}(\Omega)=k_{5} X_{2}+k_{6} \operatorname{FT}\left\{u x_{1}\right\}(\Omega),
\end{gathered}
$$

where $\operatorname{FT}\left\{x_{i}\right\}=X_{i}(\Omega)$ is the FT of the signal $x_{i}$. For the case when $u=\mu \sin (\omega t)$, we have

$$
\begin{aligned}
\Omega X_{1}(\Omega)= & -j k_{1} X_{1}(\Omega)+\frac{k_{3} \mu}{2}\left(X_{2}(\Omega+\omega)-X_{2}(\Omega-\omega)\right) \\
& +k_{2} \mu \pi(\delta(\Omega+\omega)-\delta(\Omega-\omega)), \\
\Omega X_{2}(\Omega)= & -j k_{5} X_{2}(\Omega)+\frac{k_{6} \mu}{2}\left(X_{1}(\Omega+\omega)-X_{1}(\Omega-\omega)\right) .
\end{aligned}
$$

Now, solving for $X_{1}(\Omega)$ and $X_{2}(\Omega)$, we have

$$
\begin{aligned}
& X_{1}(\Omega) \\
& =\frac{\mu}{\Omega+j k_{1}}\left[k_{2} \pi(\delta(\Omega+\omega)-\delta(\Omega-\omega))\right. \\
& \left.+\frac{k_{3}}{2}\left(X_{2}(\Omega+\omega)-X_{2}(\Omega-\omega)\right)\right], \\
& X_{2}(\Omega)=\frac{\mu k_{6}}{2\left(\Omega+j k_{5}\right)}\left[X_{1}(\Omega+\omega)-X_{1}(\Omega-\omega)\right] .
\end{aligned}
$$

Equations (33) and (34) show that variables $x_{1}, x_{2}$ have the form of low pass filters (as we commented previously). Thus, the terms $X_{2}(\Omega-\omega)$ and $X_{2}(\Omega+\omega)$ in (33) are as follows:

$$
\begin{gathered}
X_{2}(\Omega-\omega)=\frac{k_{6} \mu}{2\left(\Omega-\omega+j k_{5}\right)}\left[X_{1}(\Omega)-X_{1}(\Omega-2 \omega)\right], \\
X_{2}(\Omega+\omega)=\frac{k_{6} \mu}{2\left(\Omega+\omega+j k_{5}\right)}\left[\left(X_{1}(\Omega+2 \omega)-X_{1}(\Omega)\right)\right],
\end{gathered}
$$

and using (33) and (35), we obtain

$$
\begin{aligned}
X_{1}(\Omega)=\frac{\mu D}{\Omega+j k_{1}}[ & k_{2} \pi(\delta(\Omega+\omega)-\delta(\Omega-\omega))+\frac{\mu k_{3} k_{6}}{2} \\
& \left.\times\left(\frac{X_{1}(\Omega+2 \omega)}{2\left(\Omega+\omega+j k_{5}\right)}+\frac{X_{1}(\Omega-2 \omega)}{2\left(\Omega-\omega+j k_{5}\right)}\right)\right],
\end{aligned}
$$

where

$$
D=\frac{2\left(\left(\Omega+j k_{5}\right)^{2}-\omega^{2}\right)\left(\Omega+j k_{1}\right)}{2\left(\left(\left(\Omega+j k_{5}\right)^{2}-\omega^{2}\right)\left(\Omega+j k_{1}\right)+\mu^{2} k_{3} k_{6}\left(\Omega+j k_{5}\right)\right)} .
$$

Equation (36) has the terms $X_{1}(\Omega-2 \omega)$ and $X_{1}(\Omega+2 \omega)$, which are calculated by developing recursively (33), giving as a result

$$
\begin{aligned}
& X_{1}(\Omega-2 \omega) \\
& =\frac{\mu}{\Omega-2 \omega+j k_{1}}\left[k_{2} \pi(\delta(\Omega-\omega)-\delta(\Omega-3 \omega))\right. \\
& \left.+\frac{k_{3}}{2}\left(X_{2}(\Omega-\omega)-X_{2}(\Omega-3 \omega)\right)\right], \\
& X_{1}(\Omega+2 \omega) \\
& =\frac{\mu}{\Omega+2 \omega+j k_{1}}\left[k_{2} \pi(\delta(\Omega+3 \omega)-\delta(\Omega+\omega))\right. \\
& \left.+\frac{k_{3}}{2}\left(X_{2}(\Omega+3 \omega)-X_{2}(\Omega+\omega)\right)\right] .
\end{aligned}
$$

Equations (33) and (38) show that $X_{1}(\Omega)$ have terms which contain factors $\delta(\Omega-3 \omega), \delta(\Omega-\omega), \delta(\Omega+\omega)$, and $\delta(\Omega+3 \omega)$ and if we develop recursively $n$ times (33), then new terms of the form $\mu^{|k|} \delta(\Omega-(2 k+1) \omega) D_{k}$ for $-n \leq k \leq n$ appear in the solution of $X_{1}(\Omega)$, where $D_{k}$ is a so complex factor which depends on every parameter and variable of (32) and for $\mu<1.0$ the quotient $\left|\mu^{k}\right| /\left|D_{k}\right| \approx 0$. Therefore, when the amplitude $\mu$ is incremented, then the amplitude of the peaks $\delta(\Omega-(2 k+1) \omega)$ does too. For this reason, the numerical evidence shows that if the parameter input signal $\mu$ is incremented, then apparently new components of frequency appear in the spectrum $X_{1}(\Omega)$. These frequencies always form part of $X_{1}(\Omega)$ but with a very small amplitude. They are so small that they look like noise. 


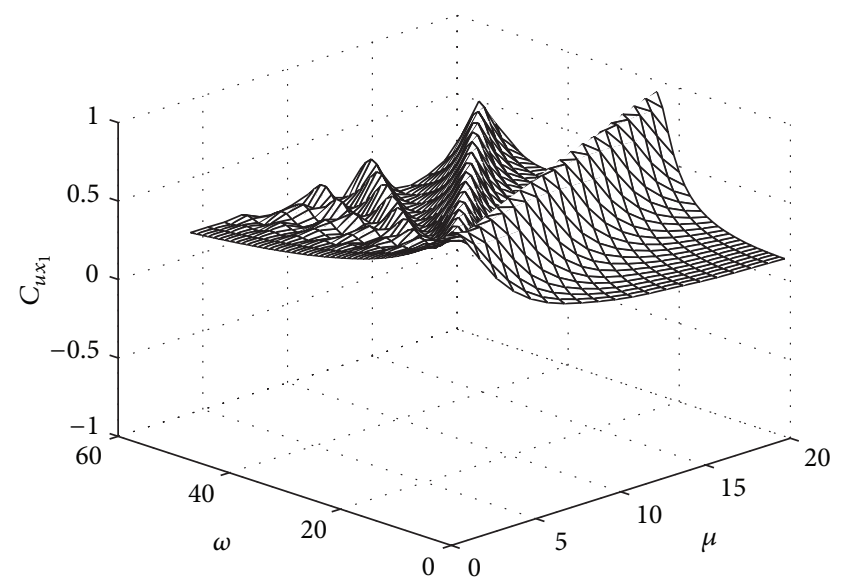

(a)

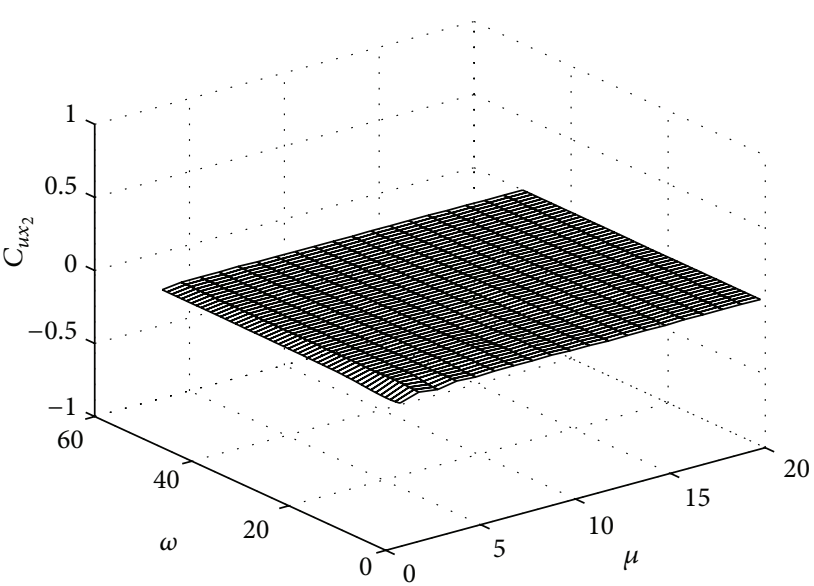

(b)

FIgURE 10: Correlation between the response of the filter (6) and the input signal $u=\mu \in(\omega t):$ (a) $C_{u x_{1}}$ and (b) $C_{u x_{2}}$.

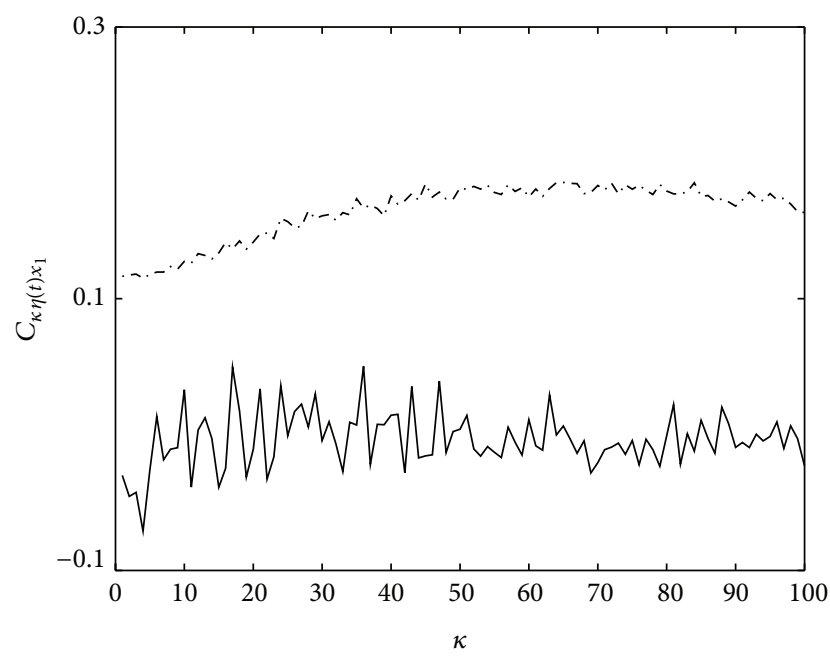

FIGURE 11: Correlation in function of the parameter of the random input signal $\kappa$. Dashed line and continuous line correspond to $C_{u x_{1}}$ and $C_{u x_{2}}$, respectively.

\section{Response to Random Signals}

Now, let $u=\kappa \eta(t)$, where $\eta(t)$ is a random signal equidistributed on the interval $[-1,1]$ and $\kappa$ is its amplitude. When $u=10 \eta(t)$ is the input signal of the filter (6), the phase space of the response is a limit cycle that has the form of a quarter moon which is shown in Figure 7(a). As a first glimpse the filter's response seems to be a periodic orbit like in the case of the sinusoidal input signal, but the time series $x_{1}(t)$ and $x_{2}(t)$, which are respectively shown in Figures $7(\mathrm{~b})$ and $7(\mathrm{c})$, are aperiodic signals.

The following remarks are pertinent.

(i) The output signal of the filter has a normal (or Gaussian) distribution when the input signal is random with uniform distribution. For the case $u=\eta(t)$, Figures 8(a) and 8(b) show the histogram and the FT of the $x_{1}$ signal, respectively, where one can see that the FT of the response has an exponential decay.

(ii) The filter presents saturation after a threshold value of the amplitude of input signal $u=\kappa \eta(t)$. Figure 9 shows the range of values $R(\kappa)=\operatorname{MAX}\left\{x_{1}\right\}-$ $\operatorname{MIN}\left\{x_{1}\right\}$ of the response $x_{1}$ versus the amplitude of the parameter $\kappa$.

To justify the exponential decay of the filter's response when it is forced with random signal, we consider $\operatorname{FT}\{u\}=K_{1}$, where $K_{1}$ is a constant which depends on $\kappa$, the amplitude of the random signal, and $x_{3} \ll \mathrm{FT}\{u\}$. Thus the following terms of (30) are $k_{2} \operatorname{FT}\{u\}=k_{2} K_{1}$ and FT $\left\{u x_{2}\right\} \approx K_{2}$, where $K_{2}$ depends on $\kappa$ too. So, the FT results are

$$
j \Omega X_{1}(\Omega) \approx-X_{1}(\Omega)-K_{2}+r K_{1},
$$

and for the magnitude of $X_{1}(\Omega)$ we have

$$
\left|X_{1}(\Omega)\right| \approx\left|\frac{r K_{1}+K_{2}}{1+\Omega^{2}}\right| .
$$

Figure $8(\mathrm{~b})$ shows the form of the curve $\left|X_{1}(\Omega)\right|$ which corresponds to (40).

\section{Correlation Coefficient}

Because a filter acts in function of the input signal, another way to characterize the effect of the input signal on the filter's response is to compute the correlation between the input and output signals. If we have time series of $N$ data, the correlation coefficient $C_{x y}$ of series $x(i)$ and $y(i)$ is defined as

$$
C_{x y}=\frac{\sum_{i=1}^{N}(x(i)-\bar{x})(y(i)-\bar{y})}{(N-1) S_{x} S_{y}},
$$

where $\bar{x}, \bar{y}$ are the means and $s_{x}, s_{y}$ are the standard deviations of the series $x(i)$ and $y(i)$, respectively. We calculate correlation coefficients for different input signals and the 


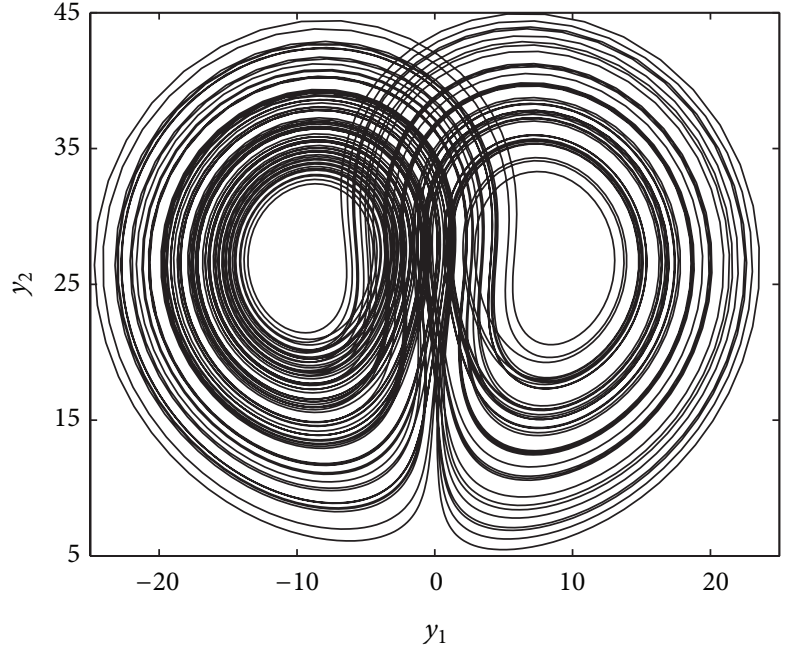

(a)

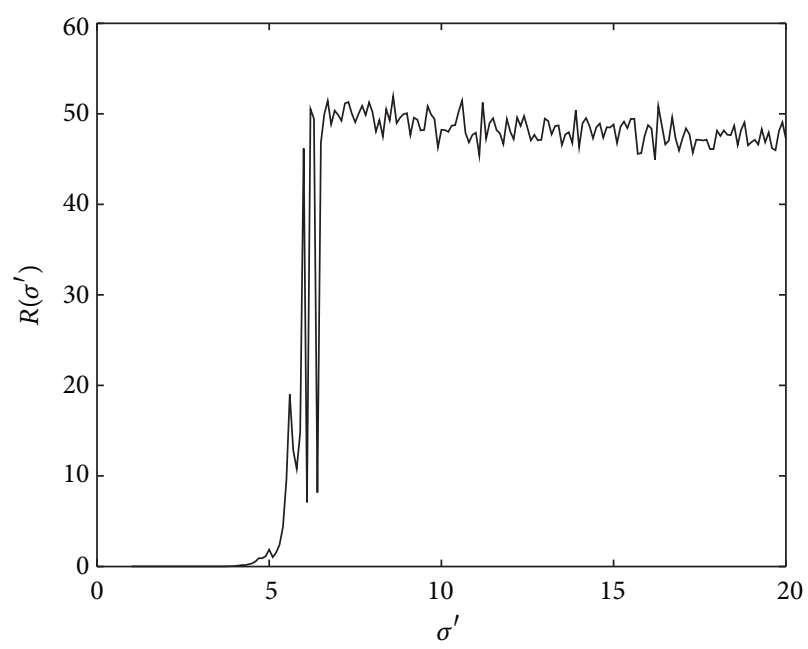

(b)

FIGURE 12: (a) Orbit of the filter (43) when the external input is $u=y_{1}$ of system (42). (b) The maximal range of response of system (43).

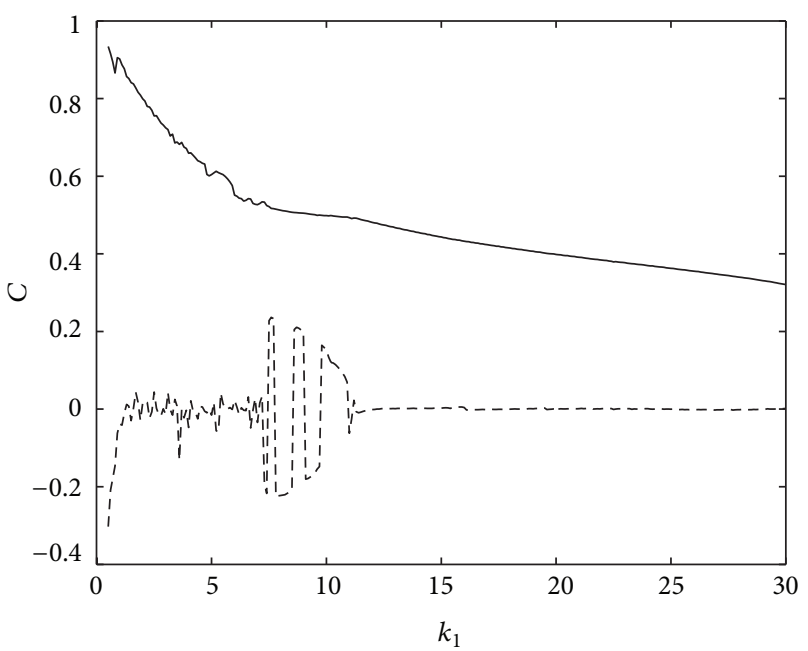

FIGURE 13: Correlation between state variables against the parameter $\kappa_{1}$. The continuous line and the dashed line correspond to $C_{y_{1} y_{2}}$, $C_{y_{1} y_{3}}$, respectively.

TABLE 1: Coefficient correlations between different input signals and the responses $x_{1}, x_{2}$ of the filter (6).

\begin{tabular}{lcc}
\hline External force $u$ & $C_{x_{1} u}$ & $C_{x_{2} u}$ \\
\hline $\sin (t)$ & 0.7455 & -0.0021 \\
The state variable $x_{1}$ of the Rössler system & 0.7453 & 0.0574 \\
$\eta(t)$ & 0.1160 & 0.0630 \\
\hline
\end{tabular}

output signals $x_{1}$ and $x_{2}$ which are given in Table 1 . The first two cases in Table 1 correspond to a periodic and chaotic signals, $C_{u x_{1}} \approx 0.745$ and $C_{u x_{2}} \approx 0.0$, and the last case considers a random signal as an input signal.

We make a further numerical study of the coefficient correlation in function of the parameters of the sinusoidal input signal. In Figure 10(a), we can see that the correlation between sinusoidal external signals is not constant and that it depends on both parameters $\mu$ and $\omega$. On the contrary, Figure 10(b) shows that the absence of correlation between the response $x_{2}$ and their respective forces is independent of almost the whole range of values of the input signal parameters.

On the other hand, the last row in Table 1 shows that $C_{u x_{1}} \approx 0.1160$ and $R_{u x_{2}} \approx 0.0630$. This means that the filter's response (6) does not have correlation when the input is a random signal. Despite the fact that the amplitude of the parameter $\kappa$ is increased, the output signal of the filter does not have correlation with input signal (random), as shown in Figure 11. Therefore, the correlation between the input random signal and its filter's response cannot be induced by incrementing the value of its amplitude. A similar result occurs for the sinusoidal signal and its response $x_{2}$.

\section{Low Pass Filters in Chaotic Systems}

The Lorenz system is a very well-known third-order chaotic system [11] which is defined as

$$
\begin{aligned}
\dot{y}_{1} & =-\sigma y_{1}+\sigma y_{2}, \\
\dot{y}_{2} & =r y_{1}-y_{2}-y_{1} y_{3} \\
& =-y_{2}+\left(r-y_{3}\right) y_{1}, \\
\dot{y}_{3} & =y_{1} y_{2}-b y_{3}=-b y_{3}+y_{2} y_{1},
\end{aligned}
$$

where $\mathbf{y}=\left(y_{1}, y_{2}, y_{3}\right)^{T}$ is the state vector and $\sigma, r$, and $b$ are parameters. Each component of system (42) is defined based on low pass filters. For example, the first equation is a linear low pass filter, where the state variables $y_{1}$ and $y_{2}$ are the input and output signals, respectively. The second and third equations comprise a two-interconnected nonlinear filter whose outputs are $y_{2}$ and $y_{3}$, and the input signal is 


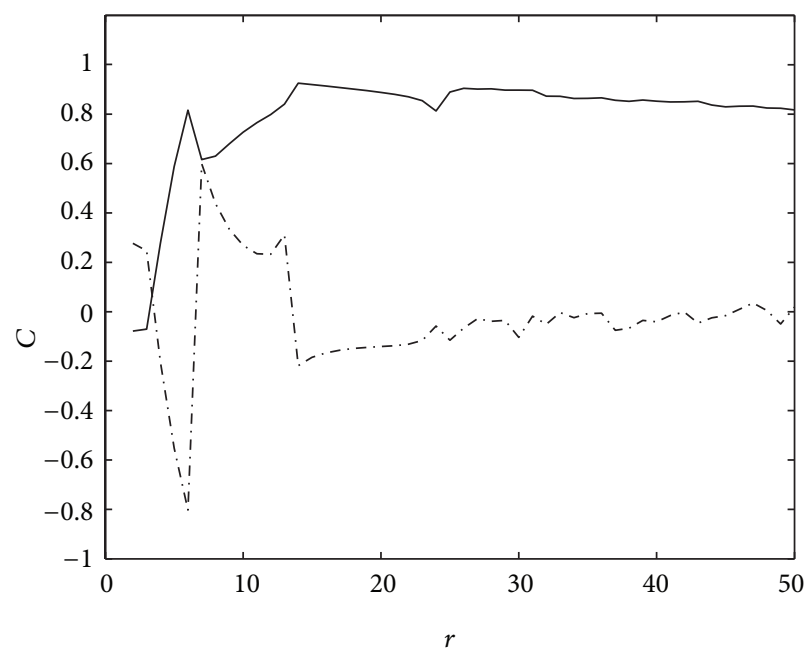

(a)

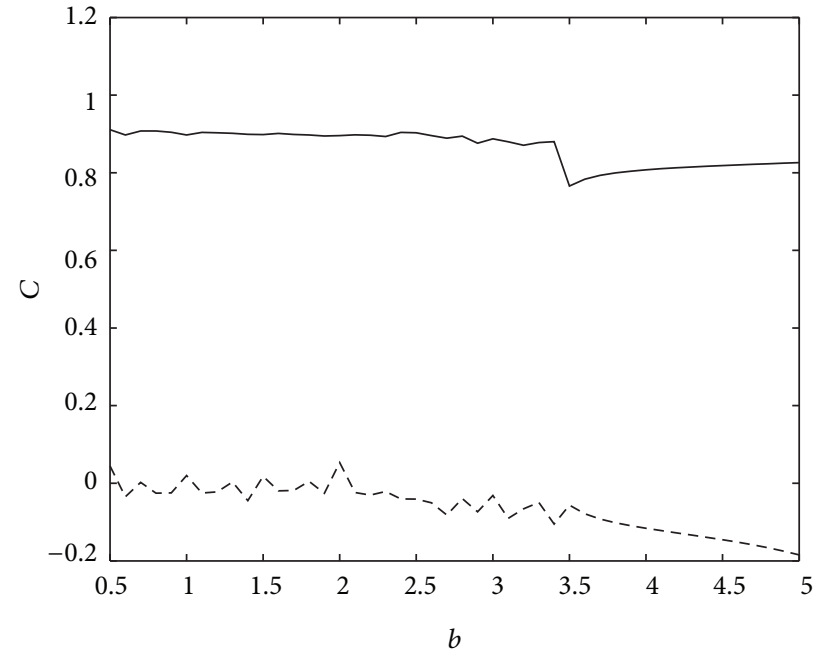

(b)

FIGURE 14: Correlations between the state variables of the Lorenz system (42) against the parameters (a) $r$ and (b) $b$. Each continuous line and dashed line correspond to $C_{y_{1} y_{2}}, C_{y_{1} y_{3}}$, respectively.

$u=y_{1}$. The two-interconnected nonlinear filter of the Lorenz system (42) can be rewritten in terms of (7) as follows:

$$
\begin{aligned}
& K=\left(\begin{array}{cc}
-1 & 0 \\
0 & -b
\end{array}\right), \quad B=\left(\begin{array}{l}
r \\
0
\end{array}\right), \\
& f(x, u)=\left(\begin{array}{l}
u y_{3} \\
u y_{2}
\end{array}\right),
\end{aligned}
$$

where $x_{1}=y_{2}, x_{2}=y_{3}$, and $u=y_{1}$. The projection of the Lorenz attractor onto the plane $\left(y_{1}, y_{2}\right)$ is shown in Figure 12(a) for the following parameter's values: $\sigma=10$, and $b=2.66$, and $r=28.0$. In order to study the response of the interconnected system (43) when the input signal is the state variable $y_{1}$, we have calculated $R(\sigma)$ which is the maximal range of values of the time series $y_{2}$. In Figure 12(b), we can see that $R \approx 0$ for $0 \leq \sigma \leq 5$; this means that the solution converges to a fixed point. For $\sigma \in[5,7]$, there is a transition period in the behavior of the filter, and for $\sigma \geq 7$ the range of values of the filter's response is approximately constant.

When calculating the correlation coefficient between the components of the two-interconnected system (43), it results that $C_{y_{1} y_{2}}=0.9022, C_{y_{1} y_{3}}=-0.0384$, and $C_{y_{2} y_{3}}=-0.0424$. The high correlation between the state variables $y_{1}$ and $y_{2}$ occurs because of $y_{1}$ is a simple low pass lineal filter for the signal $y_{2}$ without any nonlinear term included in its equation (see $\dot{y}_{1}$ in (42)). On the other hand, the time series $y_{1}$ has no correlation with $y_{3}$ which could be induced by the nonlinear term settles in the equation $\dot{y}_{3}$ of system (42). Now, we put a parameter to control the amplitude of the input signal such that $u=\kappa_{1} y_{1}$ and calculate $C_{y_{1} y_{2}}$ and $C_{y_{2} y_{3}}$ as a function of the parameter $k_{1}$. In Figure 13, it is shown that only $C_{y_{1} y_{2}}$ is affected when the value of $\kappa_{1}$ is incremented, while the absence of correlation between states $y_{2}$ and $y_{3}$ remains for all values of $\kappa_{1}$. For $\kappa_{1}>32$, the trajectories of system (43) diverge.
We have shown by means of numerical experiments in previous sections that the absence of correlation between the external force $u$ and the state variable $x_{2}$ of the filter response generally does not depend on the parameters of the input signal. Similar result occurs when the Lorenz autonomous system is considered like a two-interconnected system which is forced by the signal $u=y_{1}$. As shown in Figure 13, there exists a high correlation between the state variables $y_{1}$ and $y_{2}$, and an absence of correlation between state variables $y_{2}$ and $y_{3}$. But can the correlation between the state variables $y_{1}$ and $y_{2}$ be incremented by changing the value of the parameters of the Lorenz system? With the purpose of answering this question, we have calculated $C_{y_{1} y_{2}}, C_{y_{1} y_{3}}$ as a function of the autonomous Lorenz system parameter $r$ keeping the value of the parameter $b$ constant and vice versa. Figure 14(a) shows that for $r<15$ approximately, correlations $C_{y_{1} y_{2}}, C_{y_{1} y_{3}}$ have a transition period, and for $r \geq 15$, the correlations return to their previously showed behavior: $C_{y_{1} y_{2}} \approx 1.0$ and $C_{y_{1} y_{3}} \approx 0$. Figure 14(b) shows that the parameter $b$ does not affect the correlation between $y_{1}$ and the responses $y_{2}, y_{3}$.

If Lorenz system parameters are tuned so that the Lorenz system behaves as a filter and can be used as a slave system, then generalized synchronization of master and slave systems always appears.

\section{Conclusions}

An $n$-interconnected nonlinear system given by (6) always behaves as a filter if the condition given by Theorem 4 is satisfied. Despite of the nonlinearities in the structure of $n$-interconnected nonlinear system, its dynamical behavior does not depend on the initial conditions but it does as a function of the input signal. The nonlinear filter's response to sinusoidal input signal presents several interesting phenomena such as (i) frequency components at multiples of the input signal's frequency (ii) when the amplitude of the input 
signal increases, the number of peaks of the filter's response increases (iii) the amplitude of the filter's response falls exponentially as a function of the input signal's frequency.

The Lorenz system is described in terms of low pass filters which consists of a linear low pass filter and a twointerconnected nonlinear low pass filter. This gives us the possibility to describe all the systems that conform the Lorenz family and others with similar structure in terms of low pass filters.

In several studies of chaos synchronization, specifically in forced systems $\dot{\mathbf{x}}=f(\mathbf{x}, u), \mathbf{x} \in \mathbb{R}^{n}$, it has been found that generalized synchronization of response systems occurs for specific external signals $u$, but all these cases satisfy condition (8). Then, the general synchronization phenomenon and the phenomenon of nonlinear filter are seen to be the same. Nevertheless, condition (8), in generalized synchronization phenomenon, could not be satisfied, because of the trajectories generated with different initial conditions asymptotically could go to a different basin of attractions. This characteristic is different to the phenomenon of nonlinear filter which by definition condition (8) needs to be always satisfied if a system behaves like a nonlinear filter. Several chaotic systems have a similar structure to $n$-interconnected system, so that we conjecture that this study can help to distinguish between generalized synchronization behavior and nonlinear filtering behavior of an $n$-interconnected system.

\section{Acknowledgments}

The authors would like to thank Emanuel Rodríguez-Orozco for reading the paper and giving constructive comments. E. Jiménez-López is a doctoral fellow of CONACYT in the Graduate Program on Applied Science at UASLP, and IPICYT for the hospitality during his sojourn in DMAp-IPICYT. E. Campos-Cantón acknowledges CONACYT for the financial support through Project no. 181002.

\section{References}

[1] L. M. Pecora and T. L. Carroll, "Synchronization in chaotic systems," Physical Review Letters, vol. 64, no. 8, pp. 821-824, 1990.

[2] I. Campos-Cantón, E. Campos-Cantón, J. S. Murgúa-Ibarra, and M. E. Chavira-Rodríguez, "Secure communication system using chaotic signals," Ingeniería, Investigación y Tecnología, vol. 10, no. 1, 2009.

[3] M. G. Rosenblum, A. S. Pikovsky, and J. Kurths, "From phase to lag synchronization in coupled chaotic oscillators," Physical Review Letters, vol. 78, no. 22, pp. 4193-4196, 221997.

[4] M. G. Rosenblum, A. S. Pikovsky, and J. Kurths, "Phase synchronization of chaotic oscillators," Physical Review Letters, vol. 76, no. 11, pp. 1804-1807, 1996.

[5] V. S. Anishchenko, T. E. Vadivasova, D. È. Postnov, and M. A. Safonova, "Synchronization of chaos," International Journal of Bifurcation and Chaos in Applied Sciences and Engineering, vol. 2, no. 3, pp. 633-644, 1992.

[6] H. D. I. Abarbanel, N. F. Rulkov, and M. M. Sushchik, "Generalized synchronization of chaos: the auxiliary system approach," Physical Review E, vol. 53, no. 5, pp. 4528-4535, 1996.
[7] E. Campos, J. Urías, and N. F. Rulkov, "Multimodal synchronization of chaos," Chaos, vol. 14, no. 1, pp. 48-54, 2004.

[8] L. Ontañón-García, E. Campos-Cantón, R. Femat, I. CamposCantón, and M. Bonilla-Marín, "Multivalued synchronization by poincar coupling," Communications in Nonlinear Science and Numerical Simulation.

[9] J. S. González Salas, E. Campos Cantón, F. C. Ordaz Salazar, and I. Campos Cantón, "Forced synchronization of a self-sustained chaotic oscillator," Chaos, vol. 18, no. 2, Article ID 023136, p. 9, 2008.

[10] E. C. Cantón, J. S. C. Salas, and J. Urías, "Filtering by nonlinear systems," Chaos, vol. 18, no. 4, Article ID 043118, p. 4, 2008.

[11] E. N. Lorenz, "Deterministic nonperiodic flow," Journal of the Atmospheric Sciences, vol. 20, pp. 130-141, 1963.

[12] L. V. Turukina and A. Pikovsky, "Hyperbolic chaos in a system of resonantly coupled weakly nonlinear oscillators," Physics Letters A, vol. 375, no. 11, pp. 1407-1411, 2011. 


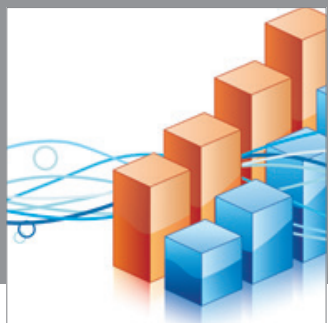

Advances in

Operations Research

mansans

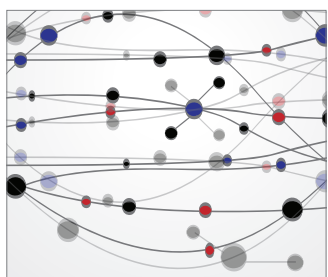

The Scientific World Journal
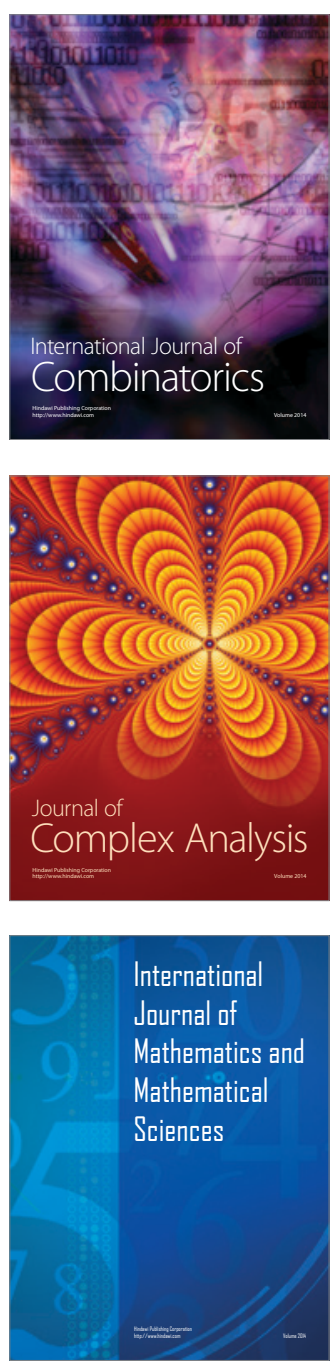
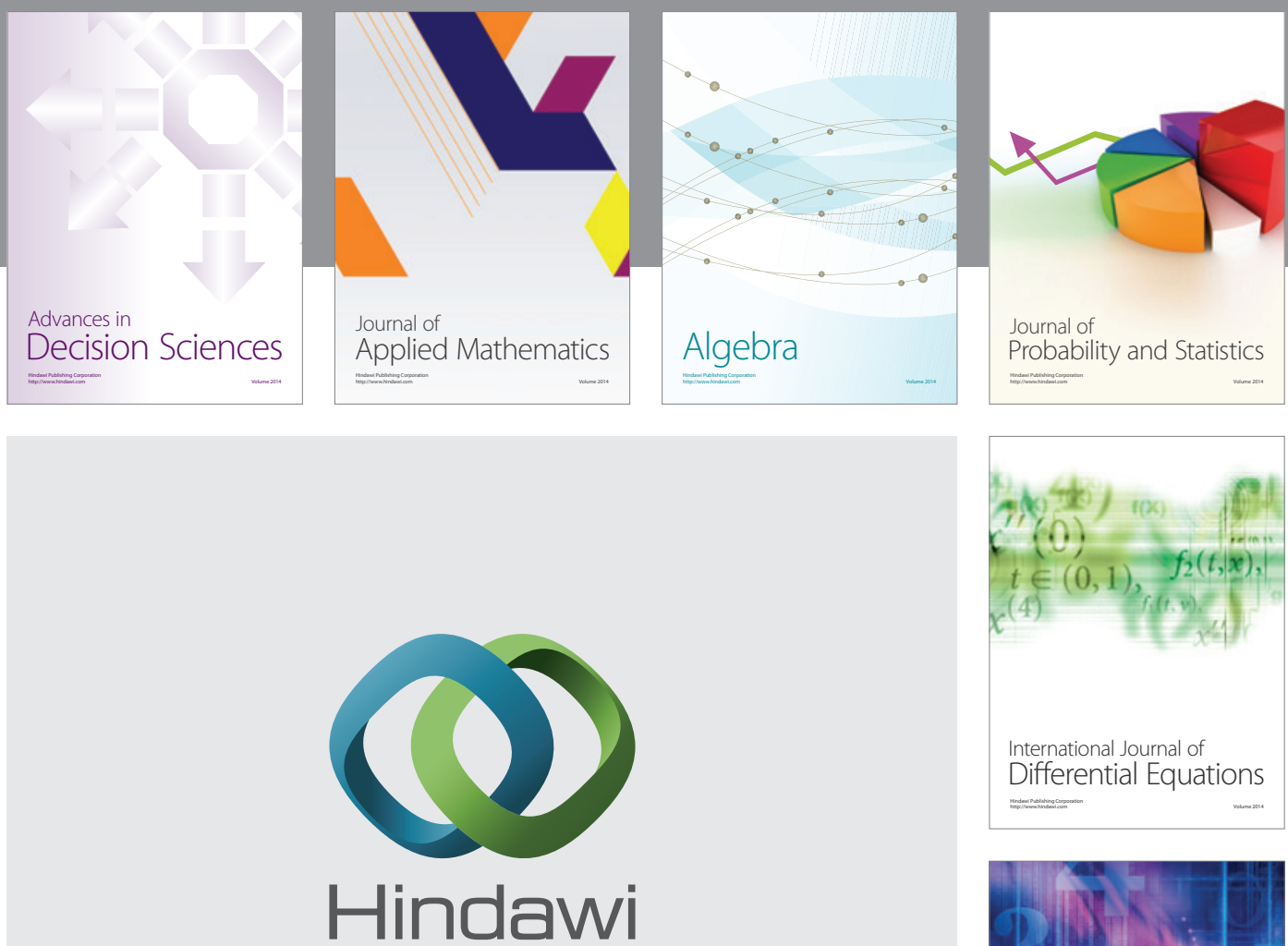

Submit your manuscripts at http://www.hindawi.com
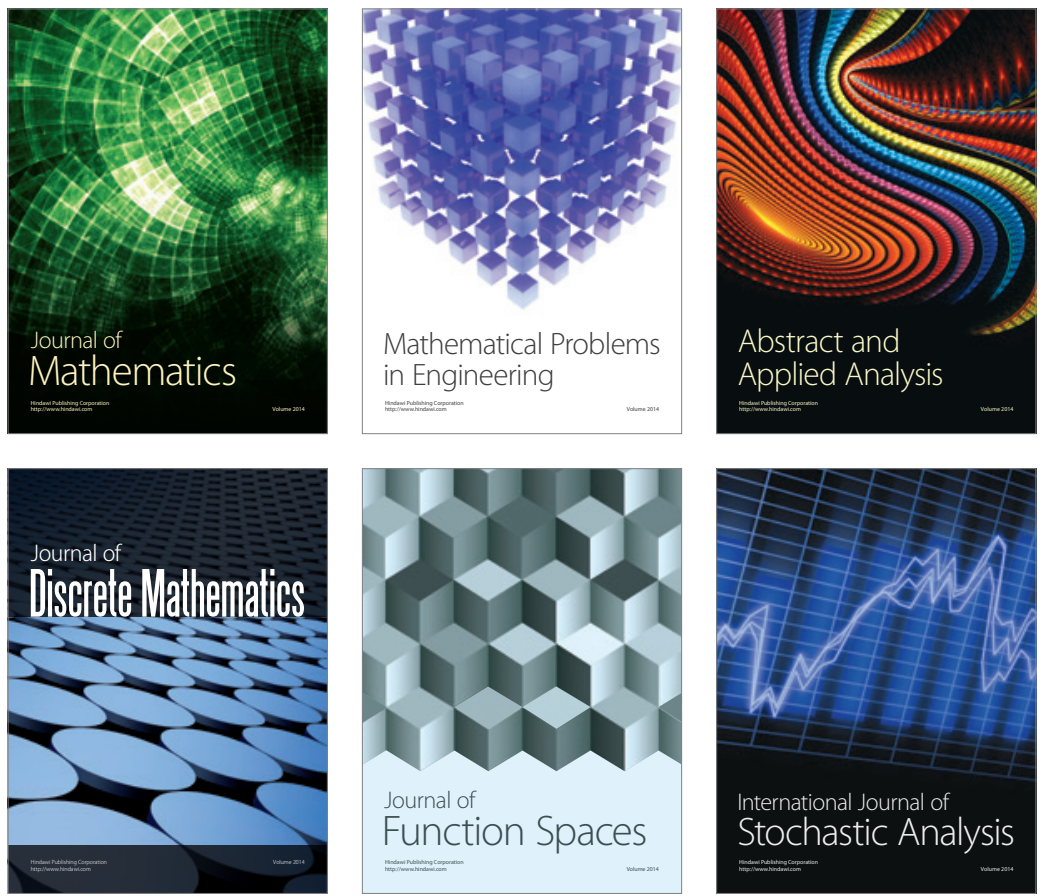

Journal of

Function Spaces

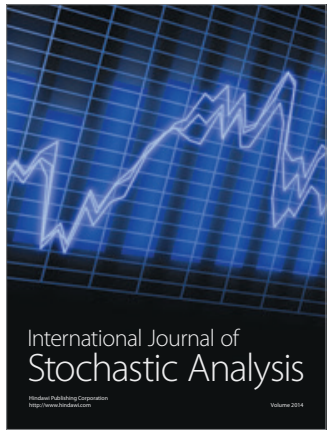

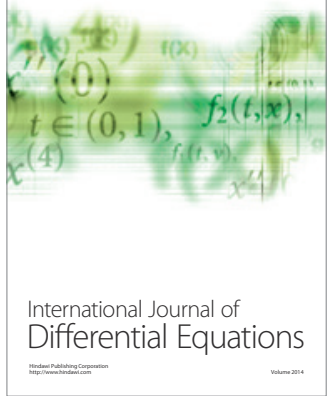
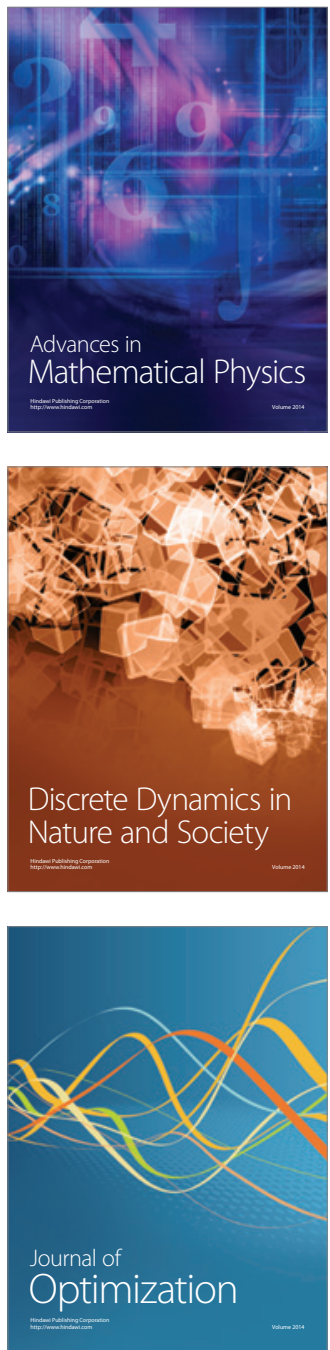